\title{
Observation of doubly-charmed B decays at LEP
}

\author{
The ALEPH Collaboration ${ }^{1}$
}

\begin{abstract}
A search for doubly-charmed B decays with both charmed mesons reconstructed is performed, using about 3.8 million hadronic $\mathrm{Z}$ decays recorded with the ALEPH detector at LEP. A clear signal is observed in the channels $\mathrm{B} \rightarrow \mathrm{D}_{\mathrm{s}} \overline{\mathrm{D}}(X)$ and $\mathrm{B} \rightarrow \mathrm{D} \overline{\mathrm{D}}(X)$ (where $\mathrm{D}$ can be either a $\mathrm{D}^{0}$, a $\mathrm{D}^{+}$or a $\mathrm{D}^{*+}$ ), providing the first direct evidence for doubly-charmed $\mathrm{B}$ decays involving no $\mathrm{D}_{\mathrm{s}}$ production. Evidence for associated $\mathrm{K}_{\mathrm{S}}^{0}$ and $\mathrm{K}^{ \pm}$production in the decays $\mathrm{B} \rightarrow \mathrm{D} \overline{\mathrm{D}}(X)$ is also presented and some candidates for completely reconstructed decays $\mathrm{B} \rightarrow \mathrm{D}_{\mathrm{S}} \overline{\mathrm{D}}(n \pi), \mathrm{B} \rightarrow \mathrm{D}_{\overline{\mathrm{D}}} \mathrm{S}_{\mathrm{S}}^{0}$ and $\mathrm{B} \rightarrow \mathrm{D}_{\bar{D} K^{ \pm}}$are observed. Furthermore, candidates for the two-body Cabibbo suppressed decays $\mathrm{B}^{0} \rightarrow \mathrm{D}^{*-} \mathrm{D}^{*+}$ and $\mathrm{B}^{-} \rightarrow \mathrm{D}^{(*) 0} \mathrm{D}^{(*)-}$ are also observed. Measurements of the corresponding branching fractions are extracted.
\end{abstract}

(To be submitted to The European Physical Journal C.)

\footnotetext{
${ }^{1}$ See next pages for the list of authors
} 


\section{The ALEPH Collaboration}

R. Barate, D. Buskulic, D. Decamp, P. Ghez, C. Goy, J.-P. Lees, A. Lucotte, E. Merle, M.-N. Minard, J.-Y. Nief,

B. Pietrzyk

Laboratoire de Physique des Particules (LAPP), IN² $P^{3}$-CNRS, F-74019 Annecy-le-Vieux Cedex,

France

R. Alemany, G. Boix, M.P. Casado, M. Chmeissani, J.M. Crespo, M. Delfino, E. Fernandez,

M. Fernandez-Bosman, Ll. Garrido, ${ }^{15}$ E. Graugès, A. Juste, M. Martinez, G. Merino, R. Miquel, Ll.M. Mir,

I.C. Park, A. Pascual, J.A. Perlas, I. Riu, F. Sanchez

Institut de Física d'Altes Energies, Universitat Autònoma de Barcelona, E-08193 Bellaterra

(Barcelona), Spain ${ }^{7}$

A. Colaleo, D. Creanza, M. de Palma, G. Gelao, G. Iaselli, G. Maggi, M. Maggi, S. Nuzzo, A. Ranieri, G. Raso,

F. Ruggieri, G. Selvaggi, L. Silvestris, P. Tempesta, A. Tricomi, ${ }^{3}$ G. Zito

Dipartimento di Fisica, INFN Sezione di Bari, I-70126 Bari, Italy

X. Huang, J. Lin, Q. Ouyang, T. Wang, Y. Xie, R. Xu, S. Xue, J. Zhang, L. Zhang, W. Zhao

Institute of High-Energy Physics, Academia Sinica, Beijing, The People's Republic of China ${ }^{8}$

D. Abbaneo, U. Becker, P. Bright-Thomas, D. Casper, M. Cattaneo, V. Ciulli, G. Dissertori, H. Drevermann, R.W. Forty, M. Frank, R. Hagelberg, J.B. Hansen, J. Harvey, P. Janot, B. Jost, I. Lehraus, P. Mato, A. Minten, L. Moneta, ${ }^{21}$ A. Pacheco, J.-F. Pusztaszeri, ${ }^{23}$ F. Ranjard, L. Rolandi, D. Rousseau, D. Schlatter, M. Schmitt, ${ }^{20}$ O. Schneider, W. Tejessy, F. Teubert, I.R. Tomalin, H. Wachsmuth

European Laboratory for Particle Physics (CERN), CH-1211 Geneva 23, Switzerland

Z. Ajaltouni, F. Badaud, G. Chazelle, O. Deschamps, A. Falvard, C. Ferdi, P. Gay, C. Guicheney, P. Henrard,

J. Jousset, B. Michel, S. Monteil, J-C. Montret, D. Pallin, P. Perret, F. Podlyski, J. Proriol, P. Rosnet

Laboratoire de Physique Corpusculaire, Université Blaise Pascal, IN ${ }^{2} P^{3}-C N R S$, Clermont-Ferrand, F-63177 Aubière, France

J.D. Hansen, J.R. Hansen, P.H. Hansen, B.S. Nilsson, B. Rensch, A. Wäänänen

Niels Bohr Institute, DK-2100 Copenhagen, Denmark ${ }^{9}$

G. Daskalakis, A. Kyriakis, C. Markou, E. Simopoulou, I. Siotis, A. Vayaki

Nuclear Research Center Demokritos (NRCD), GR-15310 Attiki, Greece

A. Blondel, G. Bonneaud, J.-C. Brient, P. Bourdon, A. Rougé, M. Rumpf, A. Valassi, ${ }^{6}$ M. Verderi, H. Videau

Laboratoire de Physique Nucléaire et des Hautes Energies, Ecole Polytechnique, IN ${ }^{2} P^{3}-C N R S$,

F-91128 Palaiseau Cedex, France

E. Focardi, G. Parrini, K. Zachariadou

Dipartimento di Fisica, Università di Firenze, INFN Sezione di Firenze, I-50125 Firenze, Italy

M. Corden, C. Georgiopoulos, D.E. Jaffe

Supercomputer Computations Research Institute, Florida State University, Tallahassee, FL 32306-

4052, USA ${ }^{13,14}$

A. Antonelli, G. Bencivenni, G. Bologna, ${ }^{4}$ F. Bossi, P. Campana, G. Capon, F. Cerutti, V. Chiarella, G. Felici,

P. Laurelli, G. Mannocchi, ${ }^{5}$ F. Murtas, G.P. Murtas, L. Passalacqua, M. Pepe-Altarelli

Laboratori Nazionali dell'INFN (LNF-INFN), I-00044 Frascati, Italy

L. Curtis, A.W. Halley, J.G. Lynch, P. Negus, V. O'Shea, C. Raine, J.M. Scarr, K. Smith, P. Teixeira-Dias, A.S. Thompson, E. Thomson 
Department of Physics and Astronomy, University of Glasgow, Glasgow G12 8QQ,United Kingdom ${ }^{10}$

O. Buchmüller, S. Dhamotharan, C. Geweniger, G. Graefe, P. Hanke, G. Hansper, V. Hepp, E.E. Kluge, A. Putzer, J. Sommer, K. Tittel, S. Werner, M. Wunsch

Institut für Hochenergiephysik, Universität Heidelberg, D-69120 Heidelberg, Germany ${ }^{16}$

R. Beuselinck, D.M. Binnie, W. Cameron, P.J. Dornan, ${ }^{2}$ M. Girone, S. Goodsir, E.B. Martin, N. Marinelli, A. Moutoussi, J. Nash, J.K. Sedgbeer, P. Spagnolo, M.D. Williams

Department of Physics, Imperial College, London SW7 2BZ, United Kingdom ${ }^{10}$

V.M. Ghete, P. Girtler, E. Kneringer, D. Kuhn, G. Rudolph

Institut für Experimentalphysik, Universität Innsbruck, A-6020 Innsbruck, Austria ${ }^{18}$

A.P. Betteridge, C.K. Bowdery, P.G. Buck, P. Colrain, G. Crawford, A.J. Finch, F. Foster, G. Hughes, R.W.L. Jones, M.I. Williams

Department of Physics, University of Lancaster, Lancaster LA1 4YB, United Kingdom ${ }^{10}$

I. Giehl, A.M. Greene, C. Hoffmann, K. Jakobs, K. Kleinknecht, G. Quast, B. Renk, E. Rohne, H.-G. Sander, P. van Gemmeren, C. Zeitnitz

Institut für Physik, Universität Mainz, D-55099 Mainz, Germany ${ }^{16}$

J.J. Aubert, C. Benchouk, A. Bonissent, G. Bujosa, J. Carr, ${ }^{2}$ P. Coyle, F. Etienne, O. Leroy, F. Motsch,

P. Payre, M. Talby, A. Sadouki, M. Thulasidas, K. Trabelsi

Centre de Physique des Particules, Faculté des Sciences de Luminy, IN $N^{2} P^{3}$-CNRS, F-13288 Marseille,

France

M. Aleppo, M. Antonelli, F. Ragusa

Dipartimento di Fisica, Università di Milano e INFN Sezione di Milano, I-20133 Milano, Italy

R. Berlich, W. Blum, V. Büscher, H. Dietl, G. Ganis, H. Kroha, G. Lütjens, C. Mannert, W. Männer, H.-G. Moser, S. Schael, R. Settles, H. Seywerd, H. Stenzel, W. Wiedenmann, G. Wolf

Max-Planck-Institut für Physik, Werner-Heisenberg-Institut, D-80805 München, Germany ${ }^{16}$

J. Boucrot, O. Callot, S. Chen, A. Cordier, M. Davier, L. Duflot, J.-F. Grivaz, Ph. Heusse, A. Höcker,

A. Jacholkowska, D.W. Kim, ${ }^{12}$ F. Le Diberder, J. Lefrançois, A.-M. Lutz, M.-H. Schune, E. Tournefier, J.-J. Veillet, I. Videau, D. Zerwas

Laboratoire de l'Accélérateur Linéaire, Université de Paris-Sud, IN² P3_CNRS, F-91405 Orsay Cedex,

France

P. Azzurri, G. Bagliesi, ${ }^{2}$ G. Batignani, S. Bettarini, T. Boccali, C. Bozzi, G. Calderini, M. Carpinelli, M.A. Ciocci, R. Dell'Orso, R. Fantechi, I. Ferrante, L. Foà, ${ }^{1}$ F. Forti, A. Giassi, M.A. Giorgi, A. Gregorio, F. Ligabue, A. Lusiani, P.S. Marrocchesi, A. Messineo, F. Palla, G. Rizzo, G. Sanguinetti, A. Sciabà, R. Tenchini, G. Tonelli, ${ }^{19}$ C. Vannini, A. Venturi, P.G. Verdini

Dipartimento di Fisica dell'Università, INFN Sezione di Pisa, e Scuola Normale Superiore, I-56010 Pisa, Italy

G.A. Blair, L.M. Bryant, J.T. Chambers, M.G. Green, T. Medcalf, P. Perrodo, J.A. Strong, J.H. von Wimmersperg-Toeller

Department of Physics, Royal Holloway \& Bedford New College, University of London, Surrey TW20 OEX, United Kingdom ${ }^{10}$

D.R. Botterill, R.W. Clifft, T.R. Edgecock, S. Haywood, P.R. Norton, J.C. Thompson, A.E. Wright Particle Physics Dept., Rutherford Appleton Laboratory, Chilton, Didcot, Oxon OX11 OQX, United Kingdom ${ }^{10}$

B. Bloch-Devaux, P. Colas, S. Emery, W. Kozanecki, E. Lançon, ${ }^{2}$ M.-C. Lemaire, E. Locci, P. Perez, J. Rander, J.-F. Renardy, A. Roussarie, J.-P. Schuller, J. Schwindling, A. Trabelsi, B. Vallage 
CEA, DAPNIA/Service de Physique des Particules, CE-Saclay, F-91191 Gif-sur-Yvette Cedex, France $^{17}$

S.N. Black, J.H. Dann, R.P. Johnson, H.Y. Kim, N. Konstantinidis, A.M. Litke, M.A. McNeil, G. Taylor Institute for Particle Physics, University of California at Santa Cruz, Santa Cruz, CA 95064, USA ${ }^{22}$

C.N. Booth, C.A.J. Brew, S. Cartwright, F. Combley, M.S. Kelly, M. Lehto, J. Reeve, L.F. Thompson Department of Physics, University of Sheffield, Sheffield S3 7RH, United Kingdom ${ }^{10}$

K. Affholderbach, A. Böhrer, S. Brandt, G. Cowan, C. Grupen, P. Saraiva, L. Smolik, F. Stephan Fachbereich Physik, Universität Siegen, D-57068 Siegen, Germany ${ }^{16}$

M. Apollonio, L. Bosisio, R. Della Marina, G. Giannini, B. Gobbo, G. Musolino

Dipartimento di Fisica, Università di Trieste e INFN Sezione di Trieste, I-34127 Trieste, Italy

J. Rothberg, S. Wasserbaech

Experimental Elementary Particle Physics, University of Washington, WA 98195 Seattle, U.S.A.

S.R. Armstrong, E. Charles, P. Elmer, D.P.S. Ferguson, Y. Gao, S. González, T.C. Greening, O.J. Hayes, H. Hu, S. Jin, P.A. McNamara III, J.M. Nachtman, ${ }^{24}$ J. Nielsen, W. Orejudos, Y.B. Pan, Y. Saadi, I.J. Scott, J. Walsh, Sau Lan Wu, X. Wu, G. Zobernig

Department of Physics, University of Wisconsin, Madison, WI 53706, USA ${ }^{11}$

\footnotetext{
${ }^{1}$ Now at CERN, 1211 Geneva 23, Switzerland.

${ }^{2}$ Also at CERN, 1211 Geneva 23, Switzerland.

${ }^{3}$ Also at Dipartimento di Fisica, INFN, Sezione di Catania, Catania, Italy.

${ }^{4}$ Also Istituto di Fisica Generale, Università di Torino, Torino, Italy.

${ }^{5}$ Also Istituto di Cosmo-Geofisica del C.N.R., Torino, Italy.

${ }^{6}$ Supported by the Commission of the European Communities, contract ERBCHBICT941234.

${ }^{7}$ Supported by CICYT, Spain.

${ }^{8}$ Supported by the National Science Foundation of China.

${ }^{9}$ Supported by the Danish Natural Science Research Council.

${ }^{10}$ Supported by the UK Particle Physics and Astronomy Research Council.

${ }^{11}$ Supported by the US Department of Energy, grant DE-FG0295-ER40896.

${ }^{12}$ Permanent address: Kangnung National University, Kangnung, Korea.

${ }^{13}$ Supported by the US Department of Energy, contract DE-FG05-92ER40742.

${ }^{14}$ Supported by the US Department of Energy, contract DE-FC05-85ER250000.

${ }^{15}$ Permanent address: Universitat de Barcelona, 08208 Barcelona, Spain.

${ }^{16}$ Supported by the Bundesministerium für Bildung, Wissenschaft, Forschung und Technologie, Germany.

${ }^{17}$ Supported by the Direction des Sciences de la Matière, C.E.A.

${ }^{18}$ Supported by Fonds zur Förderung der wissenschaftlichen Forschung, Austria.

${ }^{19}$ Also at Istituto di Matematica e Fisica, Università di Sassari, Sassari, Italy.

${ }^{20}$ Now at Harvard University, Cambridge, MA 02138, U.S.A.

${ }^{21}$ Now at University of Geneva, 1211 Geneva 4, Switzerland.

${ }^{22}$ Supported by the US Department of Energy, grant DE-FG03-92ER40689.

${ }^{23}$ Now at School of Operations Research and Industrial Engineering, Cornell University, Ithaca, NY 148533801, U.S.A.

${ }^{24}$ Now at University of California at Los Angeles (UCLA), Los Angeles, CA 90024, U.S.A.
} 


\section{Introduction}

Decays of B mesons to a charmed and an anticharmed meson plus anything are expected to occur through the b quark to c quark transitions $\overline{\mathrm{b}} \rightarrow \overline{\mathrm{c}} \mathrm{W}^{+}$, where the $\mathrm{W}^{+}$materializes as c$\overline{\mathrm{s}}$. Evidence for such decays comes mainly from experiments running at the $\Upsilon(4 S)$, which have shown $[1,2]$ evidence for inclusive $D_{s}$ production in $B$ meson decays and also evidence for exclusive twobody decays ${ }^{1} \mathrm{~B} \rightarrow \mathrm{D}_{\mathrm{s}}^{+(*)} \mathrm{D}^{-(*)}, \mathrm{D}_{\mathrm{s}}^{+(*)} \overline{\mathrm{D}}^{0(*)}$. The most recent measurement of the $\mathrm{B} \rightarrow \mathrm{D}_{\mathrm{s}} X$ decays is $\mathcal{B}\left(\mathrm{B} \rightarrow \mathrm{D}_{\mathrm{s}} X\right)=\left(12.11 \pm 0.39_{\text {stat }} \pm 0.88_{\text {syst }} \pm 1.38_{\mathcal{B}\left(\mathrm{D}_{\mathrm{s}} \rightarrow \phi \pi\right)}\right) \%$ [2]. From an analysis of the energy spectrum of the $D_{\mathrm{s}}$ mesons produced at the $\Upsilon(4 S)$, the branching fraction for the two-body component is found to be $\mathcal{B}\left(\mathrm{B} \rightarrow \mathrm{D}_{\mathrm{s}} X\right.$ (two-body) $)=(5.52 \pm 0.57 \pm 1.35 \pm 0.53) \%$ [2] and is interpreted as due to transitions $\bar{b} \rightarrow \bar{c}(c \bar{s})$. However, the mechanisms responsible for the remaining $\mathrm{D}_{\mathrm{s}}$ production at the $\Upsilon(4 S)$ have not been clearly identified and could be either $\overline{\mathrm{b}} \rightarrow \overline{\mathrm{c}}(\mathrm{c} \overline{\mathrm{s}})$ transitions or $\overline{\mathrm{b}} \rightarrow \overline{\mathrm{c}}(\mathrm{u} \overline{\mathrm{d}})$ transitions with $\mathrm{s} \overline{\mathrm{s}}$ quark popping.

Until recently, it was believed that the $c \bar{s}$ quarks would hadronize dominantly as $\mathrm{D}_{\mathrm{s}}^{+(*)}$ mesons. Therefore, the branching fraction $\overline{\mathrm{b}} \rightarrow \overline{\mathrm{c}} \mathrm{c} \overline{\mathrm{s}}$ was computed from the inclusive $\mathrm{B} \rightarrow \mathrm{D}_{\mathrm{s}} X$, $\mathrm{B} \rightarrow(\mathrm{c} \overline{\mathrm{c}}) X$ and $\mathrm{B} \rightarrow \Xi_{\mathrm{c}} X$ branching fractions, leading to $\mathcal{B}(\mathrm{b} \rightarrow \mathrm{c} \overline{\mathrm{cs}})=15.8 \pm 2.8 \% \quad[3]$. Theoretical calculations are unable to simultaneously describe this low branching fraction and the semileptonic branching fraction of the B meson [4]. It has been conjectured [5] that $\mathcal{B}(\mathrm{b} \rightarrow \mathrm{c} \overline{\mathrm{c}})$ is in fact larger and that decays $\mathrm{B} \rightarrow \mathrm{D} \overline{\mathrm{DK}}(X)$ (where $\mathrm{D}$ can be either a $\mathrm{D}^{0}$ or a $\mathrm{D}^{+}$) could contribute significantly. This might also include possible decays to orbitallyexcited $\mathrm{D}_{\mathrm{s}}$ mesons, $\mathrm{B} \rightarrow \overline{\mathrm{D}}^{(*)} \mathrm{D}_{\mathrm{s}}^{* *}$, followed by $\mathrm{D}_{\mathrm{s}}^{* *} \rightarrow \mathrm{D}^{(*)} \overline{\mathrm{K}}$. This picture is supported by the evidence for wrong-sign $\mathrm{D}$ production in $\mathrm{B}$ decays which was found recently by CLEO and yields $\mathcal{B}(\mathrm{B} \rightarrow \mathrm{D} X)=(7.9 \pm 2.2) \%[6]$.

At LEP, the high statistics and the long decay length of the $\mathrm{B}$ mesons produced allow comprehensive investigations to be made of the $\mathrm{b} \rightarrow \mathrm{c}(\overline{\mathrm{c}} \mathrm{s})$ transitions. In the analysis described below, two-body decays $\mathrm{B} \rightarrow \mathrm{D}_{\mathrm{s}}^{+(*)} \overline{\mathrm{D}}^{(*)}$ and many-body decays $\mathrm{B} \rightarrow \mathrm{D}_{\mathrm{s}}^{+} \overline{\mathrm{D}} X$ and $\mathrm{B} \rightarrow \mathrm{D} \overline{\mathrm{D}} X$ (involving no $D_{\mathrm{s}}$ and never previously seen) have been searched for by completely reconstructing two charmed mesons in the same hemisphere and trying to find a common vertex (the B decay vertex). A measurement of the corresponding branching fractions is given, covering nearly all the possibilities for doubly-charmed B decays. Candidates for completely reconstructed decays $\mathrm{B} \rightarrow \mathrm{D}_{\mathrm{s}}^{+} \overline{\mathrm{D}}(n \pi), \mathrm{B} \rightarrow \mathrm{D}_{\bar{D}} K_{\mathrm{S}}^{0}$ and $\mathrm{B} \rightarrow \mathrm{DDK}^{ \pm}$, as well as for the Cabibbo suppressed decay $\mathrm{B}^{0} \rightarrow \mathrm{D}^{*+} \mathrm{D}^{*-}$, are also presented.

\section{The ALEPH detector}

A detailed description of the ALEPH detector and of its performance can be found elsewhere $[7,8]$. Only a brief description of the properties of the apparatus relevant for this analysis is given here. Charged particles are tracked in an axial magnetic field of $1.5 \mathrm{~T}$ using a silicon vertex

\footnotetext{
${ }^{1}$ Charge-conjugate reactions are implied throughout this paper.
} 
detector (VDET), a drift chamber (ITC) and a time projection chamber (TPC). Surrounding the beam pipe, the VDET consists of two concentric layers of double-sided silicon detectors, positioned at average radii of $6.5 \mathrm{~cm}$ and $11.3 \mathrm{~cm}$, and covering $85 \%$ and $69 \%$ of the solid angle, respectively. The vertex detector has a spatial resolution of $12 \mu \mathrm{m}$ in $r \phi$ and between $12 \mu \mathrm{m}$ and $22 \mu \mathrm{m}$ for the $z$ coordinate, depending on the polar angle of the track. The ITC, at radii between $16 \mathrm{~cm}$ and $26 \mathrm{~cm}$, provides up to 8 coordinates per track in the $r \phi$ view, while the TPC measures up to 21 three-dimensional points per track at radii between $30 \mathrm{~cm}$ and $180 \mathrm{~cm}$. The combined tracking system has a transverse momentum resolution of $\sigma\left(p_{T}\right) / p_{T}=0.0006 \times p_{T} \oplus 0.005\left(p_{T}\right.$ in $\mathrm{GeV} / c)$.

In addition to tracking, the TPC is used for particle identification by measurement of the ionization energy loss associated with each charged track; it provides up to $338 \mathrm{~d} E / \mathrm{d} x$ measurements. In this paper, the $\mathrm{d} E / \mathrm{d} x$ information is considered available when more than 50 measurements are associated to a charged particle. This occurs for $82 \%$ of the tracks and this fraction is well simulated in the Monte Carlo. In the following, particle identification with energy loss is specified in term of the $\mathrm{d} E / \mathrm{d} x$ estimator defined as $\chi_{H}=\left(I_{H}-I_{m}\right) / \sigma_{H}$, where $I_{m}$ is the measured energy loss, $I_{H}$ the expected energy loss under the mass hypothesis $H$ ( $H=\pi$, $\mathrm{K}, \ldots)$ and $\sigma_{H}$ is the expected error on $I_{H}$.

Photons and $\pi^{0}$ 's are identified in the electromagnetic calorimeter (ECAL), a leadproportional chamber sandwich segmented in $0.9^{\circ} \times 0.9^{\circ}$ projective towers which are read out in three sections in depth. The energy resolution achieved is $\sigma(E) / E=0.25 / \sqrt{E / \mathrm{GeV}}$ for single $\gamma$ in hadronic jets, and about $6.5 \%$, almost independent of the energy, for $\pi^{0}$, using the kinematical constraint of the $\pi^{0}$ mass [8].

\section{Event selection}

\subsection{Data sample and outline}

This analysis uses a sample of about 3.8 million hadronic $\mathrm{Z}$ decays recorded by ALEPH in the period 1991-1995. The selection of $\mathrm{Z} \rightarrow \mathrm{q} \overline{\mathrm{q}}$ events is based on charged tracks and is described elsewhere [9]; its efficiency is $97.5 \%$. For the selected events the interaction point is reconstructed on an event-by-event basis using the constraint of the beam axis position and the size of the luminous region [10]. This interaction point is determined with an average resolution projected along the sphericity axis of the event of $85 \mu \mathrm{m}$ for b $\bar{b}$ events. Doublycharmed hadronic B decays are identified by looking for events with both a charmed and an anticharmed meson candidate in the same hemisphere originating from a common vertex (the $\mathrm{B}$ decay vertex). The charmed mesons can be either a $\mathrm{D}^{0}, \mathrm{D}^{+}, \mathrm{D}^{*+}$ or a $\mathrm{D}_{\mathrm{s}}^{+}$. They are searched for in the decay modes $\mathrm{D}^{0} \rightarrow \mathrm{K}^{-} \pi^{+}, \mathrm{D}^{0} \rightarrow \mathrm{K}^{-} \pi^{+} \pi^{-} \pi^{+}, \mathrm{D}^{+} \rightarrow \mathrm{K}^{-} \pi^{+} \pi^{+}, \mathrm{D}^{*+} \rightarrow \mathrm{D}^{0} \pi^{+}$, $\mathrm{D}_{\mathrm{s}}^{+} \rightarrow \phi \pi^{+}\left(\phi \rightarrow \mathrm{K}^{-} \mathrm{K}^{+}\right)$and $\mathrm{D}_{\mathrm{s}}^{+} \rightarrow \overline{\mathrm{K}}^{* 0} \mathrm{~K}^{+}\left(\overline{\mathrm{K}}^{* 0} \rightarrow \mathrm{K}^{-} \pi^{+}\right)$. For $\mathrm{D}^{0}$ mesons from $\mathrm{D}^{*+}$ decay, the decay mode $\mathrm{D}^{0} \rightarrow \mathrm{K}^{-} \pi^{+} \pi^{0}$ is also used. 


\subsection{Charmed meson selection}

The charmed mesons are reconstructed using all possible combinations of pion and kaon track candidates with at least one hit in the silicon vertex detector (VDET). For all the decay modes, at least two tracks are required to have VDET hits in both the $r \phi$ and $z$ views. Pion candidates are required to have a momentum greater than $0.5 \mathrm{GeV} / c$, whilst kaons are required to have a momentum greater than $1.6 \mathrm{GeV} / c$. For $\mathrm{D}^{0} \rightarrow \mathrm{K}^{-} \pi^{+} \pi^{-} \pi^{+}$, the lowest momentum pion threshold is lowered to $0.35 \mathrm{GeV} / c$, but the two highest momentum pions must satisfy $p_{\pi}>1 \mathrm{GeV} / c$. For $\mathrm{D}^{+} \rightarrow \mathrm{K}^{-} \pi^{+} \pi^{+}$, at least one of the pions must satisfy $p_{\pi}>1 \mathrm{GeV} / c$. For the decay $\mathrm{D}^{*+} \rightarrow \mathrm{D}^{0} \pi^{+}$followed by $\mathrm{D}^{0} \rightarrow \mathrm{K}^{-} \pi^{+} \pi^{0}$, the $\pi^{0}$ is selected using the algorithm described in [8] and is required to have energy $E_{\pi^{0}}>1 \mathrm{GeV}$.

The charged kaon candidates are selected using the $\mathrm{d} E / \mathrm{d} x$ information from the TPC, when available: the associated tracks are required to satisfy $-3<\chi_{\mathrm{K}}<1.5$. For $\mathrm{D}^{*+}$ and $\mathrm{D}^{0} \rightarrow \mathrm{K}^{-} \pi^{+}$, which have a lower combinatorial background, a looser cut $-3<\chi_{\mathrm{K}}<2$ is used. All pion candidates are required to satisfy $-3<\chi_{\pi}<3$, when the $\mathrm{d} E / \mathrm{d} x$ information is available.

The track combinations satisfying the above criteria are fit to a common vertex. The $\chi^{2}$ probability of the vertex fit must be larger than $0.1 \%$. Finally, the reconstructed D vertex must lie at least 3 standard deviations away from the interaction point. Since the decays $\mathrm{D}^{0} \rightarrow \mathrm{K}^{-} \pi^{+} \pi^{-} \pi^{+}$and $\mathrm{D}^{+} \rightarrow \mathrm{K}^{-} \pi^{+} \pi^{+}$suffer from a relatively high level of combinatorial background, stronger particle identification and vertexing criteria are applied. In this case, the availability of the $\mathrm{d} E / \mathrm{d} x$ information for kaons is mandatory and, except for $\mathrm{D}^{0}$ from $\mathrm{D}^{*+}$, the $\mathrm{D}$ vertex is also required to be at least $1 \mathrm{~mm}$ away from the interaction point and tracks with $p<3 \mathrm{GeV} / c$ which have a probability larger than $50 \%$ of originating from the interaction point are discarded.

The $\mathrm{D}^{*+}$ candidates are reconstructed in the channel $\mathrm{D}^{*+} \rightarrow \mathrm{D}^{0} \pi^{+}$. The difference between the reconstructed masses of the $\mathrm{D}^{0} \pi^{+}$and the $\mathrm{D}^{0}$ candidate must be within $2.5 \mathrm{MeV} / c^{2}$ of 145.4 MeV $/ c^{2}$, which corresponds to approximately 3 times the average measured resolution for this quantity. For the decays $\mathrm{D}_{\mathrm{s}}^{+} \rightarrow \phi \pi^{+}\left(\mathrm{D}_{\mathrm{s}}^{+} \rightarrow \overline{\mathrm{K}}^{* 0} \mathrm{~K}^{+}\right.$), a cut at $\pm 6 \mathrm{MeV} / c^{2}$ (resp. \pm $\left.25 \mathrm{MeV} / c^{2}\right)$ around the nominal $\phi\left(\overline{\mathrm{K}}^{* 0}\right)$ mass is applied to the reconstructed $\mathrm{K}^{+} \mathrm{K}^{-}\left(\mathrm{K}^{-} \pi^{+}\right)$ mass. For $\mathrm{D}_{\mathrm{s}}^{+} \rightarrow \overline{\mathrm{K}}^{* 0} \mathrm{~K}^{+}$, a cut $\left|\cos \theta_{K}^{*}\right|>0.6$ is also performed on the helicity angle of the $\mathrm{K}^{+}$in the rest frame of the $\overline{\mathrm{K}}^{* 0}$, to take advantage of the decay distribution.

The decays $\mathrm{D}_{\mathrm{s}}^{+} \rightarrow \phi \pi^{+}$and $\mathrm{D}_{\mathrm{s}}^{+} \rightarrow \overline{\mathrm{K}}^{* 0} \mathrm{~K}^{+}$are a potential background to $\mathrm{D}^{+} \rightarrow \mathrm{K}^{-} \pi^{+} \pi^{+}$. These are effectively removed by rejecting $\mathrm{D}^{+}$candidates where one of the pions is compatible with the $\mathrm{K}^{+}$hypothesis and a $\phi$ or $\mathrm{K}^{* 0}$ candidate satisfying the above requirements can be formed.

To be able to estimate the combinatorial background, all the candidates which form an invariant mass in the range $1.7-2.0 \mathrm{GeV} / c^{2}\left(\mathrm{D}^{0}, \mathrm{D}^{+}\right)$or $1.8-2.1 \mathrm{GeV} / c^{2}\left(\mathrm{D}_{\mathrm{s}}^{+}\right)$are selected. For $\mathrm{D}^{0} \rightarrow \mathrm{K}^{-} \pi^{+} \pi^{0}$, which has a poorer mass resolution, the mass range of the selected candidates is $1.6-2.1 \mathrm{GeV} / c^{2}$. 


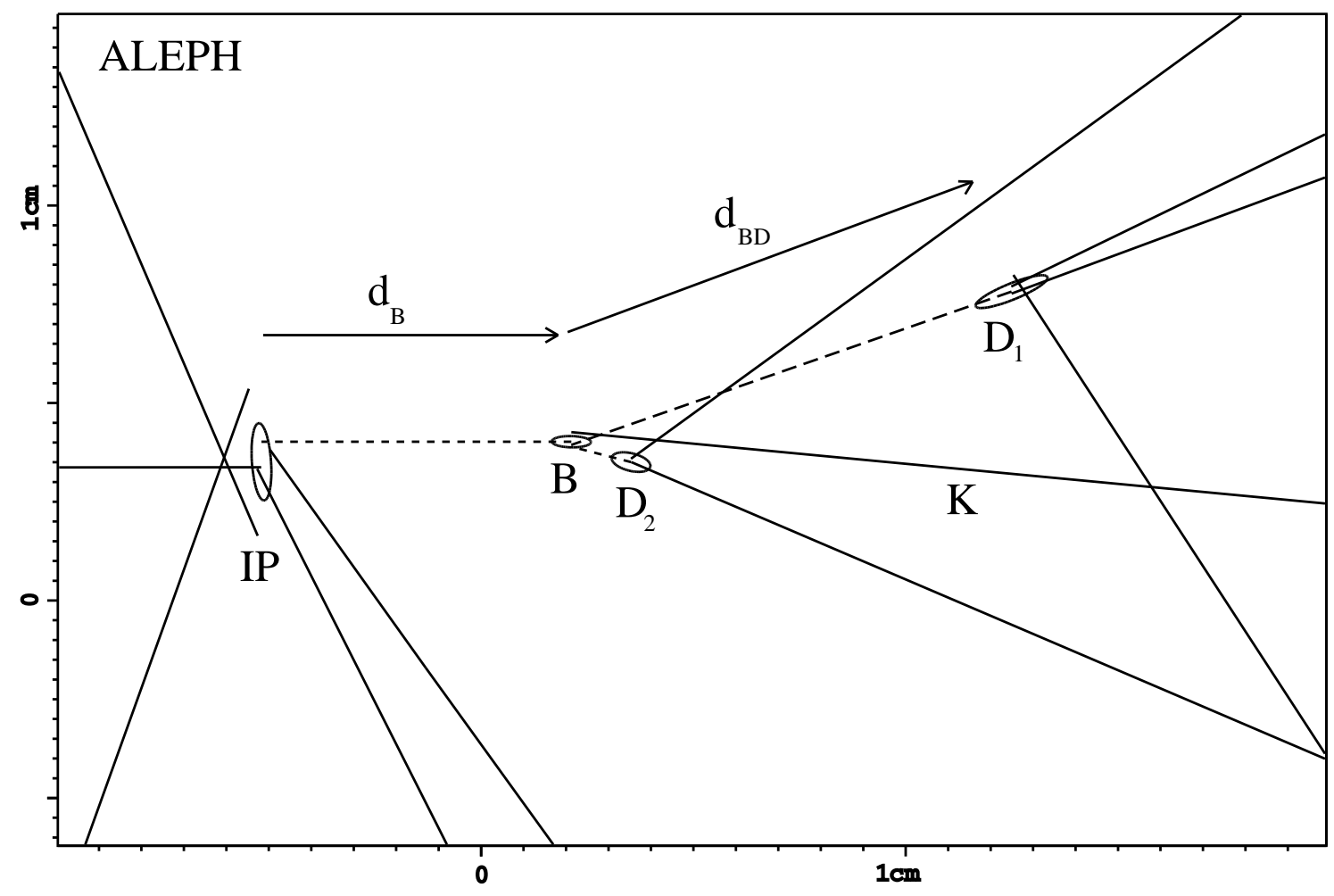

Figure 1: Display of a decay $\mathrm{B}^{0} \rightarrow \mathrm{D}^{-} \mathrm{D}^{0} \mathrm{~K}^{+}$reconstructed in the ALEPH detector (real data, event 26856/1266 from Table 11).

\subsection{Selection of doubly-charmed B decays}

A typical $\bar{D} \bar{D} X$ event reconstructed in the ALEPH detector is shown in Fig.1. To select such decays, pairs of $\mathrm{D}$ candidates are selected that belong to the same hemisphere. The two $\mathrm{D}$ candidates, denoted $\mathrm{D}_{1}$ and $\mathrm{D}_{2}$ in the following, are required to form a vertex with a probability of at least $0.1 \%$. In true $\mathrm{B} \rightarrow \mathrm{D} \overline{\mathrm{D}} X$ decays, the two $\mathrm{D}$ decay vertices must be downstream of the $\mathrm{B}$ vertex relative to the interaction point. In Fig.2, the distance $d_{\mathrm{BD}}$ between the reconstructed $\mathrm{B}$ and $\mathrm{D}$ vertices, normalised by its error $\sigma_{\mathrm{BD}}$, is displayed for simulated $\mathrm{B} \rightarrow \mathrm{D} \overline{\mathrm{D}} X$ and $\mathrm{B} \rightarrow \mathrm{DD}_{\mathrm{s}}^{* *}$ decays and for combinatorial background events, after the requirements on the $\mathrm{D}_{1} \mathrm{D}_{2}$ vertex reconstruction. The $\mathrm{D}^{+}$, which has a larger lifetime, is displayed separately from the $\mathrm{D}^{0}$ and $\mathrm{D}_{\mathrm{s}}^{+}$. Because of the larger average boost of the D's, the mean $d_{\mathrm{BD}} / \sigma_{\mathrm{BD}}$ is larger for $\mathrm{B} \rightarrow \mathrm{DD}_{\mathrm{s}}^{* *}$ events than for multibody decays $\mathrm{B} \rightarrow \mathrm{D} \overline{\mathrm{D}} X$. To maintain a good acceptance for the $\mathrm{B} \rightarrow \mathrm{D} \bar{D} X$ signal whilst rejecting the backgrounds and minimizing the model dependence of the selection efficiencies, a cut $d_{\mathrm{BD}} / \sigma_{\mathrm{BD}}>-2(>0)$ is applied on the $\mathrm{D}^{0}, \mathrm{D}_{\mathrm{s}}^{+}\left(\mathrm{D}^{+}\right)$decay length significance. The decay length significance of the $\mathrm{DD}$ vertex is also required to satisfy the condition $d_{\mathrm{B}} / \sigma_{\mathrm{B}}>-2$. Finally, a cut on the sum of the two D momenta, $p_{\mathrm{D}_{1}}+p_{\mathrm{D}_{2}}>15 \mathrm{GeV} / c$ is applied to further reduce the remaining combinatorial 
background. No requirement is made on the opposite hemisphere.

\section{Monte Carlo simulation and efficiency calculation}

In order to compute efficiencies and study physical backgrounds for the various decay channels, a Monte Carlo program based on JETSET 7.3 [11] is used. Full detector simulation is applied to Monte Carlo events which are subsequently processed through the same reconstruction program as used for real events. The energy spectra of $b$ hadrons are generated according to the Peterson et al. [12] fragmentation function. The $\mathrm{b}$ hadron properties are chosen to reproduce the most up-to-date experimental results [13]. A sample of about 3.5 million $\mathrm{Z} \rightarrow \mathrm{q} \overline{\mathrm{q}}, 1.3$ million $\mathrm{Z} \rightarrow \mathrm{b} \overline{\mathrm{b}}$ and 0.3 million $Z \rightarrow c \bar{c}$ events is used. In addition, a sample of about 100,000 events with $B$ decays forced to $\mathrm{D} \overline{\mathrm{D}}(X)$ and $\mathrm{D}$ decays forced to the modes used in this analysis is used to reduce the statistical uncertainty on the various selection efficiencies and to estimate the model dependence of those efficiencies.

To compute the efficiencies for doubly-charmed $\mathrm{B}$ decays involving one $\mathrm{D}_{\mathrm{s}}$ meson, both twobody and multibody decays are used. Multibody decays are generated using the phase-space decay scheme implemented in JETSET. The relative contribution of each process was adjusted to study the model dependence of the selection efficiencies.

In the case of decays $\mathrm{B} \rightarrow \mathrm{D} \overline{\mathrm{D}}(X)$ involving no $\mathrm{D}_{\mathrm{s}}$ meson, the contribution of the Cabibbo suppressed two-body decays $\mathrm{B} \rightarrow \mathrm{D}^{(*)} \overline{\mathrm{D}}^{(*)}$ is expected to be small; this is confirmed by existing experimental limits [14] and by the analysis described below. In the acceptance calculation for the inclusive measurement of $\mathrm{B} \rightarrow \mathrm{D} \overline{\mathrm{D}}(X)$ this contribution is therefore neglected. However, a sample of 21,000 Cabibbo suppressed two-body decays $B \rightarrow D^{(*)} \bar{D}^{(*)}$ has been simulated for specific studies concerning that mode.

Other processes contributing to $\mathrm{B} \rightarrow \mathrm{D} \overline{\mathrm{D}}(X)$ can be either multibody decays $\mathrm{B} \rightarrow \mathrm{D}^{(*)} \overline{\mathrm{D}}^{(*)} \mathrm{K}^{(*)} \quad(+n \pi)$ or two-body decays $\mathrm{B} \rightarrow \overline{\mathrm{D}}^{(*)} \mathrm{D}_{\mathrm{s}}^{* *}$ with subsequent decay of the orbitally-excited $\mathrm{D}_{\mathrm{s}}^{* *}$ state to $\mathrm{D}^{(*) 0} \mathrm{~K}^{+}$or $\mathrm{D}^{(*)+} \mathrm{K}^{0}$. Multibody decays $\mathrm{B} \rightarrow \mathrm{D}^{(*)} \overline{\mathrm{D}}^{(*)} \mathrm{K}^{(*)}(+n \pi)$ are simulated using the JETSET phase-space decay scheme mentioned above. A sample of 42,000 events with D decays forced to the channels considered in this analysis have been simulated.

Heavy Quark Effective Theory (HQET) predicts the existence and properties of four orbitally excited ( $\mathrm{P}$ Wave) $\mathrm{D}_{\mathrm{s}}^{* *}$ mesons. Two of these are expected to be narrow and have been observed [15]. Only one of these, the $\mathrm{D}_{\mathrm{s} 1}^{+}$, is expected to be produced by the weak decay process $\mathrm{W}^{+} \rightarrow \mathrm{c} \overline{\mathrm{s}}$. It has a mass of $2535 \mathrm{MeV} / c^{2}$ and is a $J^{P}=1^{+}$state, decaying dominantly to $\mathrm{D}^{*} \mathrm{~K}$. Equal statistics of decays $\mathrm{B} \rightarrow \overline{\mathrm{D}}_{\mathrm{s} 1}^{+}$and $\mathrm{B} \rightarrow \overline{\mathrm{D}}^{*} \mathrm{D}_{\mathrm{s} 1}^{+}$have been generated in the Monte Carlo simulation used here. From isospin symmetry, the $\mathrm{D}_{\mathrm{s} 1}^{+}$was assumed to decay equally to $\mathrm{D}^{* 0} \mathrm{~K}^{+}$and $\mathrm{D}^{*+} \mathrm{K}^{0}$. 

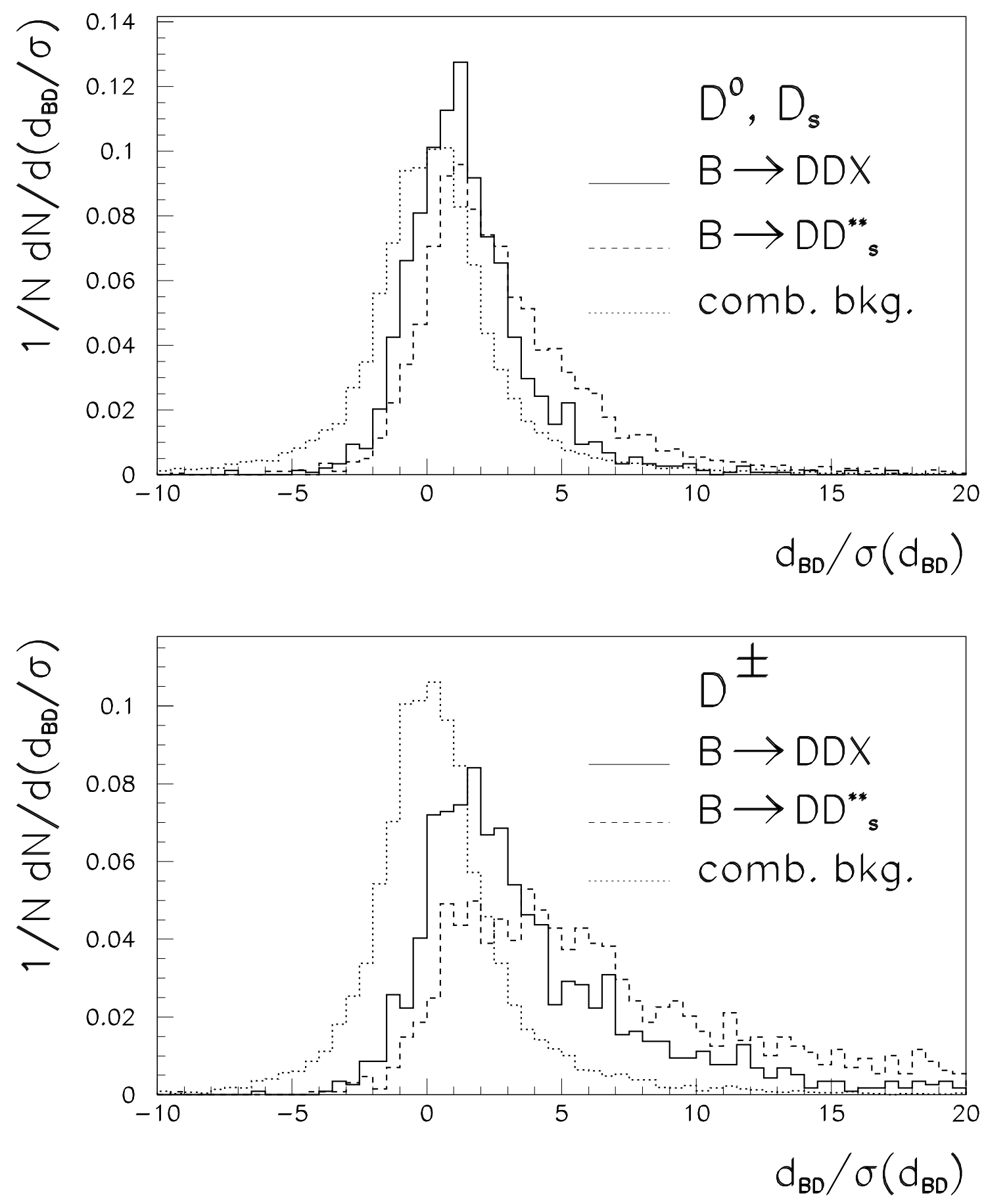

Figure 2: Distribution of the $\mathrm{D}^{0}, \mathrm{D}_{\mathrm{s}}^{+}$(top) and $\mathrm{D}^{+}$(bottom) decay length significance, relative

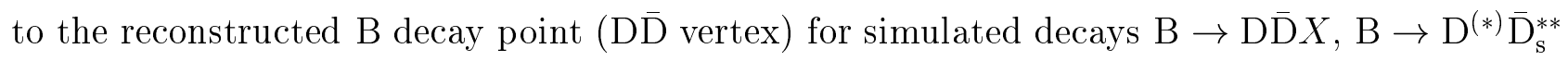
and for combinatorial background events. 


\section{Inclusive branching fractions}

\subsection{Event counting}

Evidence for decays $\mathrm{b} \rightarrow \mathrm{D} \overline{\mathrm{D}}(X)$ is obtained by histogramming the $\mathrm{D}_{1}$ vs $\mathrm{D}_{2}$ mass distributions for every possible combination of $\mathrm{D}$ and $\overline{\mathrm{D}}$ decay channels. A selection of the signals observed for a few typical channels is shown in Fig.3. The $\mathrm{D}_{1}$ vs $\mathrm{D}_{2}$ distributions, as well as their projections, are shown. The binning of the 2 dimensional mass table is chosen in order to get all the signal events into a single (central) bin. Using \pm 3 times the experimental resolution on the $\mathrm{D}$ mass peaks, the bin size is chosen to be $90 \mathrm{MeV} / c^{2}$ for $\mathrm{D}^{0} \rightarrow \mathrm{K} \pi \pi^{0}, 70$ $\mathrm{MeV} / c^{2}$ for $\mathrm{D}^{0} \rightarrow \mathrm{K} \pi$ and $50 \mathrm{MeV} / c^{2}$ for the other channels.

In each channel, the number of background events contributing to the signal bin has to be estimated. The background can be divided into two categories: the pure combinatorial background and the combination of a true $\mathrm{D}\left(\mathrm{D}_{1}\right.$ or $\left.\mathrm{D}_{2}\right)$ with combinatorial background:

$$
N_{b k g}=N\left(b_{1} b_{2}\right)+N\left(b_{1} \mathrm{D}_{2}\right)+N\left(\mathrm{D}_{1} b_{2}\right) .
$$

It was checked using Monte Carlo that the number of background events can be estimated from simple event counting averaged over symmetric sidebands around the D mass peak. For that, upper and lower sidebands regions are defined for the $\mathrm{D}_{1}$ and the $\mathrm{D}_{2}$ candidates. The width chosen for the sidebands is $180 \mathrm{MeV} / c^{2}$ for $\mathrm{D}^{0} \rightarrow \mathrm{K} \pi \pi^{0}, 70 \mathrm{MeV} / c^{2}$ for $\mathrm{D}^{0} \rightarrow \mathrm{K} \pi$ and 100 $\mathrm{MeV} / c^{2}$ for the other channels. The pure combinatorial background contribution $N\left(b_{1} b_{2}\right)$ is first estimated by averaging the content of the bins belonging both to the $D_{1}$ and to the $D_{2}$ sidebands (i.e. the corners of the 2-dimensional tables in Fig.3). The contributions $N\left(b_{1} \mathrm{D}_{2}\right)$ and $N\left(\mathrm{D}_{1} b_{2}\right)$ are then computed in a similar way for events lying at the D mass peak in one projection and in the D sidebands for the other projection, after subtracting the pure background component.

The total number of events in the signal region, the estimated background and the resulting excess are given in Table 1 . Also given in Table 1 is the sensitivity, defined as $\sum_{i, j}\left(\epsilon_{i j} \times \mathcal{B}\left(\mathrm{D}_{1} \rightarrow i\right) \times \mathcal{B}\left(\mathrm{D}_{2} \rightarrow j\right)\right)$, where $\mathcal{B}\left(\mathrm{D}_{1} \rightarrow i\right)$ and $\mathcal{B}\left(\mathrm{D}_{2} \rightarrow j\right)$ are the $\mathrm{D}$ branching fractions to modes $i$ and $j$, and $\epsilon_{i j}$ is the detection efficiency for the final state with $\mathrm{D}_{1} \rightarrow i$ and $\mathrm{D}_{2} \rightarrow j$. Typical efficiencies range from $\epsilon_{i j}=1 \%$ up to $\epsilon_{i j}=20 \%$ in the most favourable channel.

A clear signal is observed in the data, both for decays involving a $D_{s}$ and for decays involving no $D_{s}$. After summing all the decay modes and removing double counting for events involving a $\mathrm{D}^{* \pm}$, which can appear both in the $\mathrm{D}^{* \pm}$ and the $\mathrm{D}^{0}$ sections of Table 1 , excesses of $41 \pm 9 \mathrm{D}_{\mathrm{s}}^{+} \overline{\mathrm{D}}(X)$ and $76 \pm 19 \mathrm{D} \overline{\mathrm{D}}(X)$ events are observed, where $\mathrm{D}$ can be either a $\mathrm{D}^{0}$, a $\mathrm{D}^{ \pm}$or a $\mathrm{D}^{* \pm}$. The corresponding $\mathrm{D} \overline{\mathrm{D}}$ mass spectra are shown in Fig.4.

\subsection{Average $\mathrm{b}$ branching fractions}

Since measurements are available from many different $D$ decay channels, the branching fractions $\mathcal{B}\left(\mathrm{b} \rightarrow \mathrm{D}_{1} \mathrm{D}_{2}(X)\right)$ for any process of the type $\mathrm{b} \rightarrow \mathrm{D}_{1} \mathrm{D}_{2}(X)$ are extracted by maximizing the 

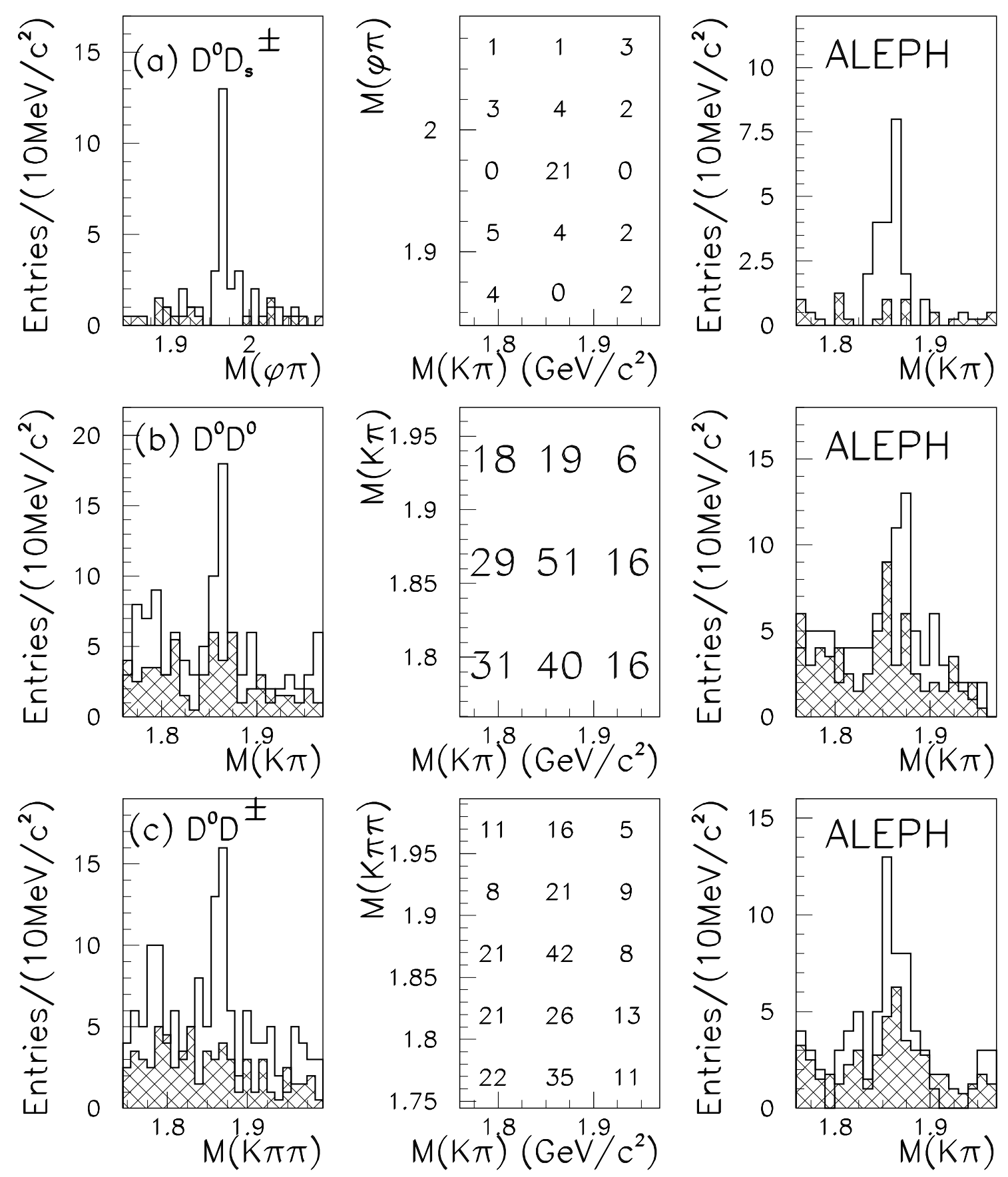

Figure 3: The $\mathrm{D}_{1}$ vs $\mathrm{D}_{2}$ mass distributions for a few typical decay channels (a) $\mathrm{B} \rightarrow \mathrm{D}^{0} \mathrm{D}_{\mathrm{s}}^{-}(X)\left(\mathrm{D}^{0} \rightarrow \mathrm{K}^{-} \pi^{+}, \mathrm{D}_{\mathrm{s}}^{-} \rightarrow \phi \pi^{-}\right) \quad(\mathrm{b}) \quad \mathrm{B} \rightarrow \mathrm{D}^{0} \overline{\mathrm{D}}^{0}(X)\left(\mathrm{D}^{0} \rightarrow \mathrm{K}^{-} \pi^{+}, \overline{\mathrm{D}}^{0} \rightarrow \mathrm{K}^{+} \pi^{-}\right) \quad$ (c) $\mathrm{B} \rightarrow \mathrm{D}^{0} \mathrm{D}^{-}(X)\left(\mathrm{D}^{0} \rightarrow \mathrm{K}^{-} \pi^{+}, \mathrm{D}^{-} \rightarrow \mathrm{K}^{+} \pi^{-} \pi^{-}\right)$. The projection along $\mathrm{D}_{1}\left(\mathrm{D}_{2}\right)$ for $\mathrm{D}_{2}\left(\mathrm{D}_{1}\right)$ inside the $\mathrm{D}$ mass window is shown as an unshaded histogram. The shaded histogram is the projection along $D_{1}\left(D_{2}\right)$ for the average of upper and lower $D_{2}\left(D_{1}\right)$ sidebands, normalised to the surface of the signal region. 


\begin{tabular}{|c|c|c|c|c|}
\hline Channel & N events & Comb. bkg. & Excess & Sensitivity $\times 10^{6}$ \\
\hline $\mathrm{D}_{\mathrm{s}} \mathrm{D}^{0}$ & 45 & $16.1 \pm 2.9$ & $28.9 \pm 7.3$ & 202 \\
\hline $\mathrm{D}_{\mathrm{s}} \mathrm{D}^{ \pm}$ & 15 & $5.1 \pm 1.4$ & $9.9 \pm 4.1$ & 146 \\
\hline $\mathrm{D}^{0} \overline{\mathrm{D}}^{0}$ & 148 & $99.1 \pm 8.3$ & $48.9 \pm 14.7$ & 493 \\
\hline $\mathrm{D}^{0} \overline{\mathrm{D}}^{ \pm}$ & 53 & $39.0 \pm 4.4$ & $14.0 \pm 8.5$ & 330 \\
\hline $\mathrm{D}^{\mp} \overline{\mathrm{D}}^{ \pm}$ & 8 & $16.8 \pm 2.5$ & $-8.8 \pm 3.8$ & 355 \\
\hline \hline $\mathrm{D}_{\mathrm{s}} \mathrm{D}^{* \pm}$ & 17 & $3.9 \pm 1.1$ & $13.1 \pm 4.3$ & 257 \\
\hline $\mathrm{D}^{0} \mathrm{D}^{* \pm}$ & 53 & $25.6 \pm 3.4$ & $27.4 \pm 8.0$ & 520 \\
\hline $\mathrm{D}^{\mp} \mathrm{D}^{* \pm}$ & 28 & $11.6 \pm 1.9$ & $16.4 \pm 5.6$ & 370 \\
\hline \hline $\mathrm{D}^{* \mp} \mathrm{D}^{* \pm}$ & 15 & $3.0 \pm 0.9$ & $12.0 \pm 4.0$ & 623 \\
\hline \hline
\end{tabular}

Table 1. The number of $\mathrm{D} \overline{\mathrm{D}}(X)$ events observed for each channel, the estimated combinatorial background, the resulting excess and the single event sensitivity. Events involving a $\mathrm{D}^{* \pm}$ decaying to $\mathrm{D}^{0} \pi^{ \pm}$also appear in the $\mathrm{DD}^{0}$ sections when the $\mathrm{D}^{0}$ from $\mathrm{D}^{* \pm}$ satisfies the inclusive $\mathrm{D}^{0}$ selection criteria.

following likelihood

$$
L=\prod_{i, j}\left(\frac{e^{-\bar{n}_{i j}} \bar{n}_{i j}^{N_{i j}}}{N_{i j} !}\right)
$$

where $N_{i j}$ is the number of events observed in the signal mass window for the D decay channels $\mathrm{D}_{1} \rightarrow i, \mathrm{D}_{2} \rightarrow j$, and $\bar{n}_{i j}$ is the expected number of events (including the combinatorial background) in that channel:

$$
\bar{n}_{i j}=N_{\mathrm{bkg}}(i, j)+2 N(\mathrm{Z}) \frac{\Gamma_{\mathrm{b} \overline{\mathrm{b}}}}{\Gamma_{\mathrm{had}}} \mathcal{B}\left(\mathrm{b} \rightarrow \mathrm{D}_{1} \mathrm{D}_{2}(X)\right) \mathcal{B}\left(\mathrm{D}_{1} \rightarrow i\right) \mathcal{B}\left(\mathrm{D}_{2} \rightarrow j\right) \epsilon_{i j}
$$

where $N_{\mathrm{bkg}}(i, j)$ is the combinatorial background measured for the channel $i, j, N(\mathrm{Z})$ is the number of hadronic $\mathrm{Z}$ events, $\mathcal{B}\left(\mathrm{D}_{1} \rightarrow i\right)$ and $\mathcal{B}\left(\mathrm{D}_{2} \rightarrow j\right)$ are the $\mathrm{D}$ branching fractions to modes $i$ and $j$, and $\epsilon_{i j}$ is the detection efficiency for the final state with $\mathrm{D}_{1} \rightarrow i$ and $\mathrm{D}_{2} \rightarrow j$. The various parameters which have been used are summarized in Table 2 . The $\mathrm{Z}$ partial width $\Gamma_{\mathrm{b} \overline{\mathrm{b}}} / \Gamma_{\text {had }}$ has been fixed to the Standard Model value. The D branching fractions have been taken from [15], as well as the relative production rate for the different species of weaklydecaying $b$ hadrons (not used here but needed in the following sections).

\subsection{Systematic uncertainties and results}

The following sources of systematic uncertainties have been considered: the simulation of the detector performance, the Monte Carlo statistics, the event counting method, the statistical uncertainty on the background, the model dependence of the selection efficiencies, the contribution from other physics processes to the D $\bar{D}$ signal and the uncertainties in the D meson 


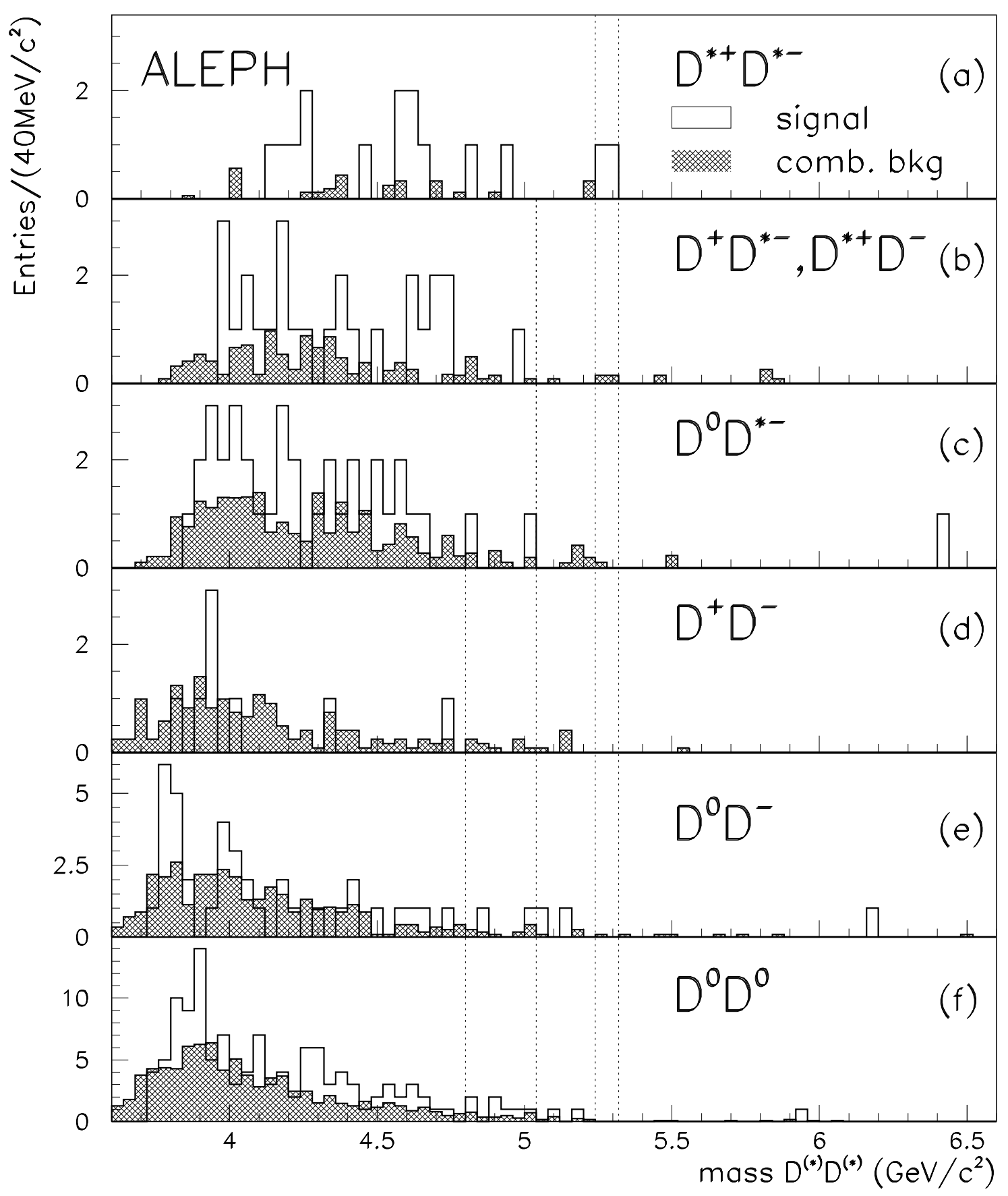

Figure 4: Unshaded histogram: the $\mathrm{D} \overline{\mathrm{D}}$ mass spectra of the selected $\mathrm{B} \rightarrow \mathrm{D} \overline{\mathrm{D}}(X)$ candidates (a) $\mathrm{D}^{*+} \mathrm{D}^{*-}$ (b) $\mathrm{D}^{ \pm} \mathrm{D}^{* \mp}$ (c) $\mathrm{D}^{0} \mathrm{D}^{*-}$ (d) $\mathrm{D}^{+} \mathrm{D}^{-}$(e) $\mathrm{D}^{0} \mathrm{D}^{-}$(f) $\mathrm{D}^{0} \overline{\mathrm{D}}^{0}$. All channels are mutually exclusive, i.e. a $\mathrm{DD}^{*+}$ event with $\mathrm{D}^{*+} \rightarrow \mathrm{D}^{0} \pi^{+}$appears only in the $\mathrm{DD}^{*+}$ histogram and not in the $\mathrm{DD}^{0}$ histogram. Shaded histogram: the $\mathrm{D} \overline{\mathrm{D}}$ mass distribution of the events in the sidebands of the $D_{1}$ or $D_{2}$ mass spectra, normalised to the expected number of combinatorial background events. Dotted lines: the $\mathrm{D} \overline{\mathrm{D}}$ mass windows corresponding to two-body decays $\mathrm{B} \rightarrow \mathrm{D}^{(*)} \overline{\mathrm{D}}^{(*)}$ with zero, one or two missed neutrals from $\mathrm{D}^{*}$ decay (cf. Section 6.4). 
branching fractions. Other systematic errors, such as the uncertainty on the b fragmentation or on the b lifetime, are expected to be small compared to the statistical errors and have been neglected.

(i) Detector performance: Differences between data and Monte Carlo in the selection efficiencies could occur through the $\mathrm{d} E / \mathrm{d} x$ requirements on the $\mathrm{K}^{ \pm}$identification and through the secondary vertex reconstruction. These effects have been thoroughly studied in previous ALEPH publications. For instance, in [16] the relative systematic errors on the individual D selection efficiencies have been estimated to be about $1 \%$ for the $\mathrm{d} E / \mathrm{d} x$ requirements and about $3 \%$ for the $\mathrm{D}$ vertex reconstruction. In the present analysis two D's and 3 vertices are reconstructed and the individual systematics on each vertex will add up. Therefore, a $10 \%$ relative systematic error due to this source is assumed in the selection of $\mathrm{B} \rightarrow \mathrm{D}_{\mathrm{s}}^{+} \overline{\mathrm{D}} X$ and $\mathrm{B} \rightarrow \mathrm{D} \overline{\mathrm{D}} X$ events.

(ii) Monte Carlo statistics: The limited Monte Carlo samples available in each decay channel introduce a relative statistical uncertainty on the selection efficiencies which ranges from about $5 \%$ for the individual $D_{1} \times D_{2}$ decay channels with the largest sensitivity up to $20 \%$ for the channels with the lowest sensitivity.

(iii) Event counting: The event counting method was tested using the $\mathrm{Z} \rightarrow \mathrm{q} \overline{\mathrm{q}}, \mathrm{Z} \rightarrow \mathrm{b} \overline{\mathrm{b}}$ and dedicated $\mathrm{B} \rightarrow \mathrm{D} \overline{\mathrm{D}}(X)$ Monte Carlo samples and comparing the number of reconstructed vs true $\mathrm{B} \rightarrow \mathrm{D} \overline{\mathrm{D}}(X)$ events. No significant bias was observed.

(iv) Background: The statistical error on the average number of background events for each channel reflects in a systematic error which is estimated by varying by $\pm 1 \sigma$ each background component in Equation (3) and repeating the analysis.

(v) Model dependence $\left(\mathrm{D}_{\mathrm{s}} \overline{\mathrm{D}}(X)\right)$ : The acceptance is larger for two-body decays $\mathrm{B} \rightarrow \mathrm{D}_{\mathrm{s}}^{(*)} \overline{\mathrm{D}}^{(*)}$ than for multibody decays $\mathrm{B} \rightarrow \mathrm{D}_{\mathrm{s}}^{(*)} \overline{\mathrm{D}}^{(*)} X$. The relative contribution of two-body decays to the total inclusive $\mathrm{D}_{\mathrm{s}}$ rate at the $\Upsilon(4 S)$ has been measured [2] to be $0.457 \pm 0.042$. This number is used here to estimate the acceptance to $\mathrm{B} \rightarrow \mathrm{D}_{\mathrm{s}} \overline{\mathrm{D}}(X)$ events. However, the \pm 0.042 error cannot be used directly, because part of the low $x_{E} \mathrm{D}_{\mathrm{s}}$ production at CLEO could be due to single $D_{s}$ production following $s \bar{s}$ popping from the sea, thus increasing the relative contribution of two-body decays in $\mathrm{B} \rightarrow \mathrm{D}_{\mathrm{s}} \overline{\mathrm{D}}(X)$. A direct measurement of multibody decays $\mathrm{B} \rightarrow \mathrm{D}_{\mathrm{s}} \overline{\mathrm{D}} X$ is presented in Section 6 of this paper. It is in agreement with the CLEO measurement and leads to measurement errors of about $13 \%$ on the relative contribution of each component. To be conservative, an error of ${ }_{-0.04}^{+0.13}$ on the relative contribution of two-body decays is assumed to estimate the corresponding systematic error on $\mathcal{B}\left(\overline{\mathrm{b}} \rightarrow \mathrm{D}_{\mathrm{s}} \overline{\mathrm{D}} X\right)$.

(vi) Model dependence (D $\overline{\mathrm{D}}(X))$ : Both multibody decays $\mathrm{B} \rightarrow \mathrm{D}^{(*)} \overline{\mathrm{D}}^{(*)} \mathrm{K}^{(*)}(n \pi)$ and twobody decays $\mathrm{B} \rightarrow \mathrm{D}_{\mathrm{s}}^{* *} \overline{\mathrm{D}}^{(*)}$ followed by $\mathrm{D}_{\mathrm{s}}^{* *} \rightarrow \mathrm{D}^{(*)} \mathrm{K}$ can contribute to the observed excess of $\mathrm{D} \overline{\mathrm{D}}(X)$ events. Because of the higher average D's boost, the acceptance is larger for $\mathrm{B} \rightarrow \mathrm{D}_{\mathrm{s}}^{* *} \overline{\mathrm{D}}^{(*)}$ than for $\mathrm{B} \rightarrow \mathrm{D}^{(*)} \overline{\mathrm{D}}^{(*)} \mathrm{K}^{(*)}(n \pi)$ events in the Monte Carlo, mainly because the rate of three-body decays $\mathrm{B} \rightarrow \mathrm{D}^{(*)} \overline{\mathrm{D}}^{(*)} \mathrm{K}$ produced in the JETSET phase-space decay scheme is low compared to the rate of many-body decays $\mathrm{B} \rightarrow \mathrm{D}^{(*)} \overline{\mathrm{D}}^{(*)} \mathrm{K} n \pi$. Therefore, the uncertainty 
on the relative contribution of each component introduces a model dependence of the selection efficiencies. In the inclusive analysis presented here, the acceptances for $\mathrm{B} \rightarrow \mathrm{D} \overline{\mathrm{D}}(X)$ are computed assuming a contribution of $50 \pm 25 \%$ from the decays $\mathrm{B} \rightarrow \overline{\mathrm{D}}^{(*)} \mathrm{D}_{\mathrm{s}}^{* *}$ to the total $\mathrm{D} \overline{\mathrm{D}}(X)$ rate. This mixture is needed to reproduce the observed $\mathrm{B} \rightarrow \mathrm{D}^{(*)} \overline{\mathrm{D}}^{(*)} \mathrm{K}$ three-body decay rate (Section 6) and also the inclusive $\bar{D} \mathrm{D}$ mass spectrum of the data, although no direct evidence for $\mathrm{D}_{\mathrm{s}}^{* *}$ production is found. The $\pm 25 \%$ error on the relative contributions of the two processes is used to estimate the systematic error corresponding to the model dependence of the selection efficiencies.

(vii) Contribution from other physics processes: The physics processes other than $\mathrm{B} \rightarrow \mathrm{D} \overline{\mathrm{D}} X$ which could contribute to the observed excess of $\mathrm{D} \overline{\mathrm{D}}$ events are either genuine $\mathrm{Z} \rightarrow \mathrm{b} \overline{\mathrm{b} g}$, $c \overline{\mathrm{c} g}$ events with both quarks in the same hemisphere, or events involving the materialization of a heavy quark pair from a radiated gluon, $g \rightarrow c \bar{c}, b \bar{b}$. No such events from $Z \rightarrow b \bar{b} g$ or $g \rightarrow b \bar{b}$ is selected from the whole Monte Carlo sample. From one selected event out of about 900,000 $\mathrm{Z} \rightarrow \mathrm{c} \overline{\mathrm{c}}$ Monte Carlo events, the contribution of $\mathrm{Z} \rightarrow \mathrm{c} \overline{\mathrm{c}} \mathrm{g}$ to the $\mathrm{D} \overline{\mathrm{D}}(X)$ signal is estimated to be smaller than 2.3 events at $90 \%$ confidence level. The contribution of $g \rightarrow c \bar{c}$ is suppressed by the cuts on the $\mathrm{D}$ decay length and by the cuts on the sum of the two D momenta. One DD pair from $\mathrm{g} \rightarrow \mathrm{c} \overline{\mathrm{c}}$ is selected out of the 3.5 million $\mathrm{Z} \rightarrow \mathrm{q} \overline{\mathrm{q}}$ Monte Carlo events, with a DD mass of $7.3 \mathrm{GeV} / c^{2}$, i.e. much larger than the B mass. From this event and using the most precise published measurement of $\mathrm{g} \rightarrow \mathrm{c} \overline{\mathrm{c}}$ [17], the contribution of $\mathrm{g} \rightarrow \mathrm{c} \overline{\mathrm{c}}$ is estimated to be smaller than 7.8 events at $90 \%$ confidence level (over the whole D $\bar{D}$ mass range) and smaller than 4.6 events for $m(\mathrm{D} \overline{\mathrm{D}})<5.4 \mathrm{GeV} / c^{2}$. In the following, both $\mathrm{Z} \rightarrow c \overline{\mathrm{c}}$ and $\mathrm{g} \rightarrow \mathrm{c} \overline{\mathrm{c}}$ contributions have been neglected when extracting the inclusive b branching fractions to $\mathrm{D} \overline{\mathrm{D}}(X)$.

(viii) $D$ meson branching fractions: All the $\mathrm{D}$ branching fractions are normalised to $\mathrm{D}^{0} \rightarrow \mathrm{K}^{-} \pi^{+}, \mathrm{D}^{+} \rightarrow \mathrm{K}^{-} \pi^{+} \pi^{+}$and $\mathrm{D}_{\mathrm{s}}^{+} \rightarrow \phi \pi^{+}$. The errors quoted in Table 2 are from [15]. They are used to estimate the corresponding systematic errors on $\mathcal{B}\left(\overline{\mathrm{b}} \rightarrow \mathrm{D}_{\mathrm{s}} \overline{\mathrm{D}}(X)\right)$ and $\mathcal{B}(\overline{\mathrm{b}} \rightarrow \mathrm{D} \overline{\mathrm{D}}(X))$.

The branching fractions measured for the average mixture of $b$ hadrons produced at LEP are summarized in Table 3, where the first error is statistical, the second is the sum of all systematic errors except those from the D branching fractions and the last one is the systematic error due to the uncertainties on the $\mathrm{D}$ meson branching fractions. The relative contribution of each source of systematic error is detailed in Table 4 for the sum of all decays to $D_{\mathrm{s}} \overline{\mathrm{D}}(X)$ and the sum of all decays to $\mathrm{D}^{0} \overline{\mathrm{D}}(X)$.

\section{Search for exclusive decays}

\subsection{Introduction}

Exclusive decays are searched for by looking for additional tracks originating from the DD vertex. In order to ensure precise vertex reconstruction, only tracks with a least one VDET hit 


\begin{tabular}{c|c|}
\hline Parameter & Value \\
\hline$\Gamma_{\mathrm{b} \bar{b}} / \Gamma_{\text {had }}$ & $21.7 \%$ \\
$N(\mathrm{Z} \rightarrow \mathrm{q} \overline{\mathrm{q}})$ & $3,838,156$ \\
$\mathrm{Z} \rightarrow \mathrm{q} \overline{\mathrm{q}}$ selection efficiency & $97.5 \%$ \\
$\mathcal{B}\left(\mathrm{D}_{\mathrm{s}} \rightarrow \phi \pi\right) \times \mathcal{B}\left(\phi \rightarrow \mathrm{K}^{+} \mathrm{K}^{-}\right)$ & $1.77 \pm 0.45 \%$ \\
$\mathcal{B}\left(\mathrm{D}^{0} \rightarrow \mathrm{K}^{+} \pi^{-}\right)$ & $3.83 \pm 0.12 \%$ \\
$\mathcal{B}\left(\mathrm{D}^{-} \rightarrow \mathrm{K}^{+} \pi^{-} \pi^{-}\right)$ & $9.1 \pm 0.6 \%$ \\
$\mathcal{B}\left(\mathrm{D}_{\mathrm{s}} \rightarrow \mathrm{K}^{* 0} \mathrm{~K}^{ \pm}\right) / \mathcal{B}\left(\mathrm{D}_{\mathrm{s}} \rightarrow \phi \pi\right)$ & $0.93 \pm 0.09$ \\
$\mathcal{B}\left(\mathrm{D}^{0} \rightarrow \mathrm{K}^{+} \pi^{-} \pi^{+} \pi^{-}\right) / \mathcal{B}\left(\mathrm{D}^{0} \rightarrow \mathrm{K}^{+} \pi^{-}\right)$ & $1.97 \pm 0.10$ \\
$\mathcal{B}\left(\mathrm{D}^{0} \rightarrow \mathrm{K}^{+} \pi^{-} \pi^{0}\right) / \mathcal{B}\left(\mathrm{D}^{0} \rightarrow \mathrm{K}^{+} \pi^{-}\right)$ & $3.62 \pm 0.24$ \\
$\mathrm{f}_{\mathrm{B}_{\mathrm{d}}^{0}}=\mathrm{f}_{\mathrm{B}^{ \pm}}$ & $37.8 \pm 2.2 \%$ \\
$\mathrm{f}_{\mathrm{B}_{\mathrm{s}}^{0}}$ & $11.2 \pm 1.9 \%$ \\
\hline
\end{tabular}

Table 2. The parameters used in the calculation of branching fractions.

\begin{tabular}{|c|c|}
\hline Channel & $\mathcal{B}(\%)$ \\
\hline $\mathrm{b} \rightarrow \mathrm{D}^{0} \mathrm{D}_{\mathrm{s}}^{-}(X)$ & $9.1_{-1.8}^{+2.0}{ }_{-1.2}^{+1.3}{ }_{-1.9}^{+3.1}$ \\
$\mathrm{~b} \rightarrow \mathrm{D}^{+} \mathrm{D}_{\mathrm{s}}^{-}(X)$ & $4.0_{-1.4}^{+1.7} \pm 0 .{ }_{-0.9}^{+1.4}$ \\
\hline $\mathrm{Sum} \mathrm{b} \rightarrow \mathrm{D}^{0} \mathrm{D}_{\mathrm{s}}^{-}, \mathrm{D}^{+} \mathrm{D}_{\mathrm{s}}^{-}(X)$ & $13.1_{-2.2}^{+2.6}{ }_{-1.6}^{+1.8}{ }_{-2.7}^{+4.4}$ \\
\hline $\mathrm{b} \rightarrow \mathrm{D}^{0} \overline{\mathrm{D}}^{0}(X)$ & $5.1_{-1.4}^{+1.6}{ }_{-1.1}^{+1.2} \pm 0.3$ \\
$\mathrm{~b} \rightarrow \mathrm{D}^{0} \mathrm{D}^{-}, \mathrm{D}^{+} \overline{\mathrm{D}}^{0}(X)$ & $2.7_{-1.3}^{+1.5}{ }_{-0.9}^{+1.0} \pm 0.2$ \\
$\mathrm{~b} \rightarrow \mathrm{D}^{+} \mathrm{D}^{-}(X)$ & $<0.9 \%$ at $90 \%^{\mathrm{C} . \mathrm{L}}$. \\
\hline $\mathrm{Sum} \mathrm{b} \rightarrow \mathrm{D}^{0} \overline{\mathrm{D}}^{0}, \mathrm{D}^{0} \mathrm{D}^{-}, \mathrm{D}^{+} \overline{\mathrm{D}}^{0}(X)$ & $7.8_{-1.8}^{+2.0}{ }_{-1.5}^{+1.7}{ }_{-0.4}^{+0.5}$ \\
\hline $\mathrm{b} \rightarrow \mathrm{D}^{*+} \mathrm{D}_{\mathrm{s}}^{-}(X)$ & $3.3_{-0.9}^{+1.0} \pm 0 .{ }_{-0.7}^{+1.1}$ \\
$\mathrm{~b} \rightarrow \mathrm{D}^{*+} \overline{\mathrm{D}}^{0}, \mathrm{D}^{0} \mathrm{D}^{*-}(X)$ & $3.0_{-0.8}^{+0.9}{ }_{-0.5}^{+0.7} \pm 0.2$ \\
$\mathrm{~b} \rightarrow \mathrm{D}^{*+} \mathrm{D}^{-}, \mathrm{D}^{+} \mathrm{D}^{*-}(X)$ & $2.5_{-0.9}^{+1.0}{ }_{-0.5}^{+0.6} \pm 0.2$ \\
\hline $\mathrm{b} \rightarrow \mathrm{D}^{*+} \mathrm{D}^{*-}(X)$ & $1.2_{-0.3}^{+0.4} \pm 0.2 \pm 0.1$ \\
\hline
\end{tabular}

Table 3. Summary of the different branching fractions measured in this analysis. The first error is statistical, the second one is the sum of all systematic errors except those from the $\mathrm{D}$ branching fractions, and the last one is the systematic error due to the uncertainty on the different $\mathrm{D}$ branching fractions. The modes involving a $\mathrm{D}^{*+}$ (lowest part of the table) are also included in the upper part results as a subsample of the modes involving a $\mathrm{D}^{0}$ or a $\mathrm{D}^{+}$. 


\begin{tabular}{|c|c|c|}
\hline & $\mathrm{b} \rightarrow \mathrm{D}_{\mathrm{s}}^{ \pm} \mathrm{D}^{0}(X), \mathrm{D}_{\mathrm{s}}^{ \pm} \mathrm{D}^{\mp}(X)$ & $\mathrm{b} \rightarrow \mathrm{D}^{0} \overline{\mathrm{D}}^{0}(X), \mathrm{D}^{0} \mathrm{D}^{\mp}(X)$ \\
\hline Combinatorial background & ${ }_{-5}^{+6}$ & ${ }_{-15}^{+17}$ \\
Monte Carlo statistics & \pm 4 & \pm 3 \\
Model & ${ }_{-5}^{+2}$ & ${ }_{-7}^{+9}$ \\
Detector & ${ }_{-9}^{+11}$ & ${ }_{-9}^{+11}$ \\
D branching fractions & ${ }_{-21}^{+34}$ & \pm 6 \\
\hline
\end{tabular}

Table 4. Relative systematic errors in percent of the $\mathcal{B}(\overline{\mathrm{b}} \rightarrow \mathrm{D} \overline{\mathrm{D}}(X))$ measurement, for the sum of $\overline{\mathrm{b}} \rightarrow \mathrm{D}_{\mathrm{s}}^{+} \overline{\mathrm{D}}^{0}(X), \mathrm{D}_{\mathrm{s}}^{+} \mathrm{D}^{-}(X)$ and the sum of $\overline{\mathrm{b}} \rightarrow \mathrm{D}^{0} \overline{\mathrm{D}}^{0}(X), \mathrm{D}^{0} \mathrm{D}^{+}(X)$ decays.

in both the $r \phi$ and $z$ projections are considered. From the $\mathrm{D}$ and $\overline{\mathrm{D}}$ tracks, the $\mathrm{B}$ decay vertex is reconstructed and a pseudo B track is created, using the direction of the $\mathrm{D}$ and $\overline{\mathrm{D}}$ momentum sum. A common vertex is then made between this pseudo B track and every additional track with momentum $p>500 \mathrm{MeV} / c$. This vertex has to be either $1 \mathrm{~mm}$ or 3 standard deviations downstream from the interaction point (with a minimum of $600 \mu \mathrm{m}$ ), with a $\chi^{2}$ probability of at least $0.1 \%$.

A search for additional $\mathrm{K}_{\mathrm{S}}^{0}$ decaying to $\pi^{+} \pi^{-}$is also performed in the $\mathrm{D} \overline{\mathrm{D}}$ hemisphere. The $\mathrm{K}_{\mathrm{S}}^{0}$ 's are identified using the algorithm described in [8]. They must have a momentum greater than $1 \mathrm{GeV} / c$ and a reconstructed mass within $15 \mathrm{MeV} / c^{2}$ of the nominal $\mathrm{K}_{\mathrm{S}}^{0}$ mass. The $\mathrm{K}_{\mathrm{S}}^{0}$ decay vertex must be located at least $1 \mathrm{~cm}$ downstream of the $\mathrm{D} \overline{\mathrm{D}}$ vertex with respect to the interaction point and its $\chi^{2}$ probability must be at least $0.1 \%$. Finally, a common vertex between the $\mathrm{D}$, the $\overline{\mathrm{D}}$ and the the $\mathrm{K}_{\mathrm{S}}^{0}$ is formed and its $\chi^{2}$ probability is required to be higher than $0.1 \%$.

\subsection{Decays $\mathrm{B} \rightarrow \mathrm{D}_{\mathrm{s}}^{+} \overline{\mathrm{D}}(X)$}

In this section, the branching fractions for the two-body decay $\mathrm{B} \rightarrow \mathrm{D}_{\mathrm{s}}^{(*)+} \overline{\mathrm{D}}^{(*)}$ (Fig.5a) and for the many-body decays $\mathrm{B} \rightarrow \mathrm{D}_{\mathrm{s}}^{+} \overline{\mathrm{D}} X$ (Fig.5b) are measured separately. Only the decay mode $\mathrm{D}_{\mathrm{s}} \rightarrow \phi \pi$, which has a high efficiency and a low combinatorial background, is used. Among the 39 events selected, only 37 are compatible with a $\mathrm{B}_{\mathrm{d}}^{0}$ or $\mathrm{B}^{+}$hypothesis $\left(m\left(\mathrm{D}_{\mathrm{s}}^{+} \overline{\mathrm{D}}\right)<5.32 \mathrm{GeV} / c^{2}\right)$, for an estimated combinatorial background of $5.5 \pm 1.3$ events. Ten events have additional tracks from the $\mathrm{D} \overline{\mathrm{D}}$ vertex; all the additional tracks are either compatible with a $\pi^{ \pm}$hypothesis or have no $\mathrm{d} E / \mathrm{d} x$ measurement available. The reconstructed $\mathrm{D}_{\mathrm{s}}^{+} \overline{\mathrm{D}}\left(n \pi^{ \pm}\right)$mass distribution is shown in Fig. 6 for the different topologies $(n=0, n \geq 1)$ and for the sum. Nine events are reconstructed at the $\mathrm{B}$ mass: six fully reconstructed two-body decays, one $\mathrm{B}^{0} \rightarrow \mathrm{D}_{\mathrm{s}}^{+} \overline{\mathrm{D}}^{0} \pi^{-}$, one $\mathrm{B}^{0} \rightarrow \mathrm{D}_{\mathrm{s}}^{+} \mathrm{D}^{*-} \pi^{+} \pi^{-}$and one $\mathrm{B}^{0} \rightarrow \mathrm{D}_{\mathrm{s}}^{+} \overline{\mathrm{D}}^{0} \pi^{-} \pi^{+} \pi^{-}$candidate. This is the first indication of completely reconstructed multibody decays $\mathrm{B}^{0} \rightarrow \mathrm{D}_{\mathrm{s}}^{+} \overline{\mathrm{D}}^{0}+n \pi^{ \pm}(n \geq 1)$.

The two-body decays $\mathrm{B} \rightarrow \mathrm{D}_{\mathrm{s}}^{(*)+} \overline{\mathrm{D}}^{(*)}$ can be distinguished from the multibody decays $\mathrm{B} \rightarrow \mathrm{D}_{\mathrm{s}}^{(*)+} \overline{\mathrm{D}}^{(*)} X$ on the basis of both the $\mathrm{D}_{\mathrm{s}}^{+} \overline{\mathrm{D}}$ mass distribution and the lack of additional 
(a)

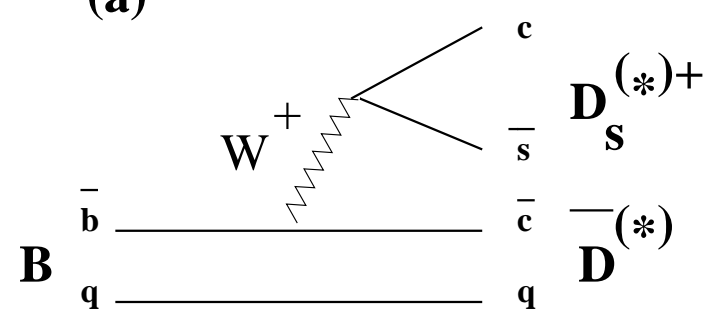

(b)

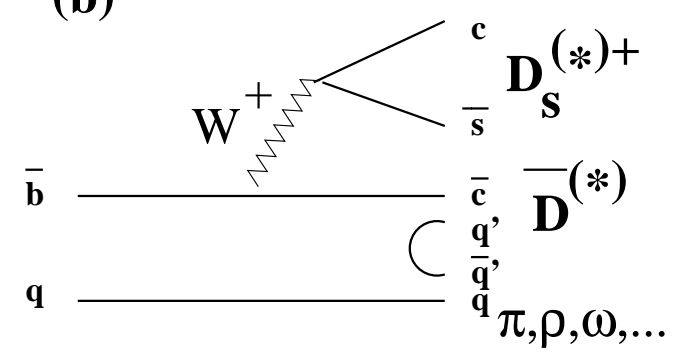

(c)

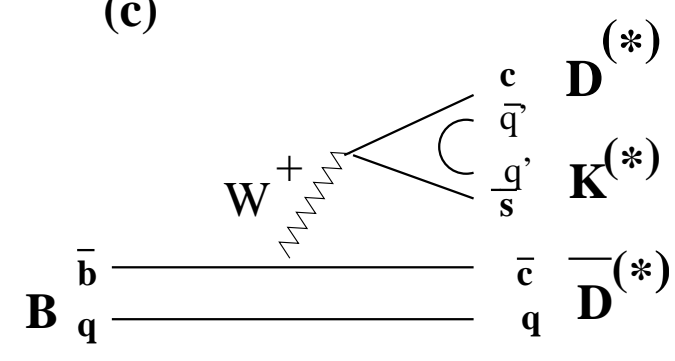

(e)

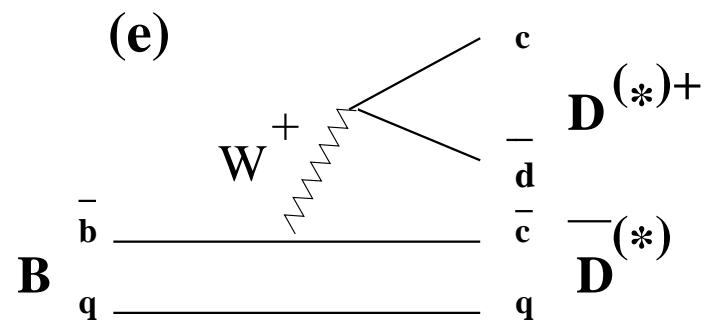

(d)

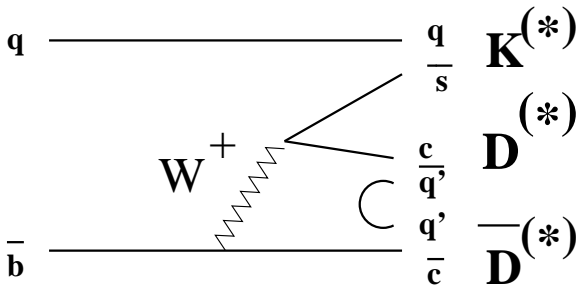

Figure 5: The different diagrams expected to contribute to two-body and three-body decays $\mathrm{B} \rightarrow \mathrm{D} \overline{\mathrm{D}}(X)$ studied in this paper $(\mathrm{a}) \mathrm{B} \rightarrow \mathrm{D}_{\mathrm{s}}^{(*)+} \overline{\mathrm{D}}^{(*)}$ (two-body) (b) $\mathrm{B} \rightarrow \mathrm{D}_{\mathrm{s}}^{(*)+} \overline{\mathrm{D}}^{(*)} \pi, \rho, \omega, \ldots$ (three-body) (c) $\mathrm{B} \rightarrow \mathrm{D}^{(*)+} \overline{\mathrm{D}}^{(*)} \mathrm{K}^{(*)}$ (three-body, external spectator) (d) $\mathrm{B} \rightarrow \mathrm{D}^{(*)+} \overline{\mathrm{D}}^{(*)} \mathrm{K}^{(*)}$ (three-body, internal spectator) (e) $\mathrm{B} \rightarrow \mathrm{D}^{(*)+} \overline{\mathrm{D}}^{(*)}$ (two-body, Cabibbo suppressed) (f) $\mathrm{B}^{0} \rightarrow \mathrm{D}^{(*) 0} \overline{\mathrm{D}}^{(*) 0}$ (two-body, W exchange).

(f)

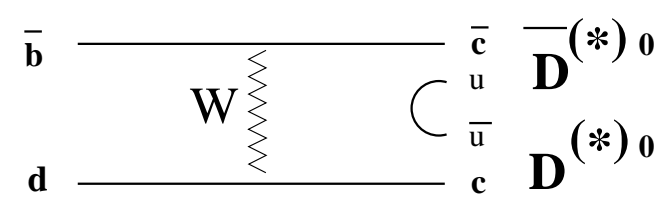


charged tracks at the $\mathrm{D} \overline{\mathrm{D}}$ vertex. In the following, events inside the two-body allowed $\mathrm{D}_{\mathrm{s}}^{+} \overline{\mathrm{D}}$ mass region $\left(4.80<m\left(\mathrm{D}_{\mathrm{s}}^{+} \overline{\mathrm{D}}^{0}, \mathrm{D}_{\mathrm{s}}^{+} \mathrm{D}^{-}\right)<5.32 \mathrm{GeV} / c^{2}\right.$ or $5.04<m\left(\mathrm{D}_{\mathrm{s}}^{+} \mathrm{D}^{*-}\right)<5.32 \mathrm{GeV} / c^{2}$, cf Fig.6a), with no additional tracks at the $\mathrm{D} \overline{\mathrm{D}}$ vertex, are classified as two-body decays (events in the upper part of the mass spectrum in Fig.6b), while other events (lower part of the mass spectum in Fig.6b plus all events with $\mathrm{D}_{\mathrm{s}}^{+} \mathrm{D} n \pi^{ \pm}<5.32 \mathrm{GeV} / c^{2}$ in Fig.6c) are classified as multibody decays. From Monte Carlo studies, it has been checked that the fraction of wrongly assigned events is less than $3 \%$ and can therefore be neglected, given the statistical errors. A total of 16 events are observed in the two-body class for an estimated combinatorial background of $1.7 \pm 0.7$ events. These events are used to estimate the following two-body branching fractions:

$$
\begin{aligned}
& \mathcal{B}\left(\mathrm{B}^{0} \rightarrow \mathrm{D}_{\mathrm{s}}^{(*)+} \mathrm{D}^{(*)-}\right)=\left(5.0_{-1.9}^{+2.9}{ }_{-1.0}^{+1.1}{ }_{-1.0}^{+1.7}\right) \% \\
& \mathcal{B}\left(\mathrm{B}^{+} \rightarrow \mathrm{D}_{\mathrm{s}}^{(*)+} \overline{\mathrm{D}}^{0(*)}\right)=\left(6.2_{-2.3}^{+3.0}{ }_{-1.0}^{+1.1}{ }_{-1.3}^{+2.1}\right) \% .
\end{aligned}
$$

Averaging over $\mathrm{B}^{0}$ and $\mathrm{B}^{ \pm}$one gets for the two-body decay modes

$$
\mathcal{B}\left(\mathrm{B} \rightarrow \mathrm{D}_{\mathrm{s}}^{(*)+} \overline{\mathrm{D}}^{(*)}\right)=\left(5.6_{-1.5}^{+2.1}{ }_{-0.8}^{+0.9}{ }_{-1.1}^{+1.9}\right) \% .
$$

The first error is statistical. The second is the systematic error resulting from detector modelling, Monte Carlo statistics, uncertainty on the combinatorial background and uncertainty on the fraction of $\mathrm{B}^{0}$ and $\mathrm{B}^{ \pm}$produced at the $\mathrm{Z}$. The third error results from the uncertainty on the different $\mathrm{D}$ branching fractions and is dominated by the uncertainty on $\mathcal{B}\left(\mathrm{D}_{\mathrm{s}}^{+} \rightarrow \phi \pi^{+}\right)$. This result is in good agreement with previous measurements of the same quantity $[1,2]$.

The multibody branching fraction is computed in the same way. Twenty-one $\mathrm{D}_{\mathrm{s}}^{+} \overline{\mathrm{D}} X$ events are observed in the multibody class for an estimated combinatorial background of $5.2 \pm 1.2$ events. To estimate the average $\mathrm{B}^{0}$ and $\mathrm{B}^{+}$many-body branching fraction $\mathcal{B}\left(\mathrm{B} \rightarrow \mathrm{D}_{\mathrm{s}}^{(*)+} \overline{\mathrm{D}}^{(*)} X\right)$, the contribution from possible decays $\mathrm{B}_{\mathrm{s}}^{0} \rightarrow \mathrm{D}_{\mathrm{s}}^{ \pm} \stackrel{(-)}{\mathrm{D}} X$ must be subtracted. A $\mathrm{B}_{\mathrm{s}}^{0}$ can decay either to $\mathrm{D}_{\mathrm{s}}^{-} \mathrm{D} \overline{\mathrm{K}}(X)$ or to $\mathrm{D}_{\mathrm{s}}^{+} \overline{\mathrm{D}} \mathrm{K}(X)$. Therefore, a reasonable guess is to assume that $\mathcal{B}\left(\mathrm{B}_{\mathrm{s}}^{0} \rightarrow \mathrm{D}_{\mathrm{s}}^{ \pm} \stackrel{(-)}{\mathrm{D}} X\right)=(2 \pm 1) \times \mathcal{B}\left(\mathrm{B} \rightarrow \mathrm{D}_{\mathrm{s}}^{(*)+} \overline{\mathrm{D}}^{(*)} X\right)$, where the \pm 1 error accounts for possible differences in the hadronisation of the $\bar{c} s$ pair (spectator quarks) and the $\bar{c}$ pair (quarks from the $\mathrm{W}$ ), and for phase space effects. With this assumption, and neglecting any possible contribution from b-baryon decays, the fraction $\rho_{s}$ of events from $\mathrm{B}_{\mathrm{s}}^{0}$ decays in the multibody $\mathrm{D}_{\mathrm{s}}^{+} \overline{\mathrm{D}} X$ sample is

$$
\rho_{s}=\frac{(2 \pm 1) \times \mathrm{f}_{\mathrm{B}_{\mathrm{s}}^{0}}}{(2 \pm 1) \times \mathrm{f}_{\mathrm{B}_{\mathrm{s}}^{0}}+\mathrm{f}_{\mathrm{B}_{\mathrm{d}}^{0}}+\mathrm{f}_{\mathrm{B}^{ \pm}}}=\left(22.9_{-10.4}^{+8.6}\right) \% .
$$

Subtracting the $\mathrm{B}_{\mathrm{s}}^{0}$ contribution and correcting for the $\mathrm{D}$ branching fractions and the multibody decay selection efficiencies, one gets

$$
\mathcal{B}\left(\mathrm{B} \rightarrow \mathrm{D}_{\mathrm{s}}^{(*)+} \overline{\mathrm{D}}^{(*)} X\right)=\left(9.4_{-3.1}^{+4.0}{ }_{-1.8}^{+2.2}{ }_{-1.6}^{+2.6}\right) \% .
$$

These results are consistent with the fully inclusive results of Section 5, but can be used to extract the fraction of two-body decays with smaller error:

$$
\frac{\mathcal{B}\left(\mathrm{B} \rightarrow \mathrm{D}_{\mathrm{s}}^{(*)+} \overline{\mathrm{D}}^{(*)}(\text { two }- \text { body })\right)}{\mathcal{B}\left(\mathrm{B} \rightarrow \mathrm{D}_{\mathrm{s}}^{(*)+} \overline{\mathrm{D}}^{(*)}(X)\right)}=(37 \pm 13) \% \text {. }
$$


Finally, a search for decays $\mathrm{B}_{\mathrm{s}}^{0} \rightarrow \mathrm{D}_{\mathrm{s}}^{+} \overline{\mathrm{D}} \mathrm{K}(X)$ is performed, looking for events with one additional $K_{S}^{0}$ correlated to the $D_{\mathrm{s}}$ and the $\mathrm{D}$. For this search, the main background is from the correlation between a genuine $\mathrm{B}^{0}$ or $\mathrm{B}^{+}$decaying to $\mathrm{D}_{\mathrm{s}}^{+} \overline{\mathrm{D}} X$ and a $\mathrm{K}_{\mathrm{S}}^{0}$ from fragmentation. To study this background, $\mathrm{K}_{\mathrm{S}}^{0} \rightarrow \pi^{+} \pi^{-}$are searched for in the $\mathrm{B}$ hemisphere using a sample of 199 completely reconstructed $\mathrm{B}^{0}$ and $\mathrm{B}^{+}$mesons decaying to $\overline{\mathrm{D}}^{(*)}+\mathrm{n} \pi$ or $\mathrm{D}_{\mathrm{s}}^{+} \overline{\mathrm{D}}$. The fraction of events with a reconstructed fragmentation $\mathrm{K}_{\mathrm{S}}^{0}$ is measured to be $(4.0 \pm 1.4) \%$ for $p\left(\mathrm{~K}^{0}\right)>1 \mathrm{GeV} / c$ and $(0.5 \pm 0.5) \%$ for $p\left(\mathrm{~K}^{0}\right)>3 \mathrm{GeV} / c$, leading to an expected contribution of $0.8 \pm 0.3$ events $\left(p\left(\mathrm{~K}^{0}\right)>1 \mathrm{GeV} / c\right)$ or $0.1 \pm 0.1$ events $\left(p\left(\mathrm{~K}^{0}\right)>3 \mathrm{GeV} / c\right)$ among the 21 $\mathrm{D}_{\mathrm{s}}^{+} \overline{\mathrm{D}} X$ events. Selecting $\mathrm{K}_{\mathrm{S}}^{0} \rightarrow \pi^{+} \pi^{-}$decays with the criteria of Section 6.1 , three events with an associated $\mathrm{K}_{\mathrm{S}}^{0}$ are found. All events are kinematically compatible with the three-body decay hypothesis $\mathrm{B}_{\mathrm{s}}^{0} \rightarrow \mathrm{D}_{\mathrm{s}}^{(*) \pm} \mathrm{D}^{(*) \mp} \mathrm{K}^{0}$ where one or more neutrals from $\mathrm{D}_{\mathrm{s}}^{* \pm} \rightarrow \mathrm{D}_{\mathrm{s}}^{ \pm} \gamma$ or $\mathrm{D}^{* \pm} \rightarrow \mathrm{D}^{ \pm} \pi^{0}$ has been missed. This hypothesis is also supported by the fact that in all three events a charged $\mathrm{D}^{(*) \pm}$ is found, while the background from fragmentation $\mathrm{K}_{\mathrm{S}}^{0}$ would also give $\mathrm{D}^{0} \mathrm{~K}_{\mathrm{S}}^{0}$ correlations. Two of the events involve a $\mathrm{K}_{\mathrm{S}}^{0}$ with momentum $p\left(\mathrm{~K}^{0}\right)>3 \mathrm{GeV} / c$ and the probability that both of them are from fragmentation is smaller than $0.5 \%$.

\subsection{Decays $\mathrm{B} \rightarrow \mathrm{D} \overline{\mathrm{DK}}(X)$}

\subsubsection{Evidence for associated $K$ production}

To check that the observed $\mathrm{D} \overline{\mathrm{D}} X$ signal is indeed due to decays $\mathrm{B} \rightarrow \mathrm{D} \overline{\mathrm{DK}}(n \pi)$, associated $\mathrm{K}$ production has been searched for in the selected sample. The $\mathrm{K}_{\mathrm{S}}^{0}$ 's are selected as described in Section 6.1. The charged K's are selected among the tracks found at the DD vertex (Section 6.1 ) on the basis of the $\mathrm{d} E / \mathrm{d} x$ measurement in the TPC. To ensure a good $\pi / \mathrm{K}$ separation, the $\mathrm{K}$ momentum is required to be greater than $1.6 \mathrm{GeV} / c$ and the $\mathrm{d} E / \mathrm{d} x$ estimator for the $\mathrm{K}$ hypothesis is required to satisfy $\chi_{K}<1$. Unambiguous low momentum K's $\left(0.5<p_{\mathrm{K}}<\right.$ $0.9 \mathrm{GeV} / c)$ are also selected requiring $\left|\chi_{K}\right|<2,\left|\chi_{\pi}\right|>2$ and $\chi_{\pi}-\chi_{K}>1.5$. Removing events where the reconstructed DD̄K mass is above the B meson mass $\left(m(\mathrm{DDK})>5.32 \mathrm{GeV} / c^{2}\right)$ and counting events in the signal region with the same technique as in Section 5.1, the results summarized in Table 5 are obtained. A clear improvement of the signal over background ratio is seen when adding the requirement of an associated K: $43 \%$ of the signal events satisfy this requirement, compared to only $12 \%$ of the combinatorial background (off peak) events. The average efficiencies for reconstructing the $\mathrm{K}$ in Monte Carlo three-body decays $\mathrm{B} \rightarrow \mathrm{D} \overline{\mathrm{DK}}$ where both D's have been reconstructed is $20.8 \pm 1.5 \%$ for $\mathrm{K}^{0}$ and $41.2 \pm 2 \%$ for $\mathrm{K}^{ \pm}$.

The reconstructed mass of the selected $\mathrm{D}^{0} \overline{\mathrm{D}}^{0} \mathrm{~K}, \mathrm{D}^{0} \mathrm{D}^{-} \mathrm{K}$ or $\mathrm{D}^{+} \mathrm{D}^{-} \mathrm{K}$ events is shown in Fig.7a. This can be compared to the spectrum expected for simulated three-body decays $\mathrm{B} \rightarrow \mathrm{D}^{(*)} \overline{\mathrm{D}}^{(*)} \mathrm{K}$ (Fig.7b). Here, the decays $\mathrm{D}^{*+} \rightarrow \mathrm{D}^{0} \pi^{+}$are not reconstructed and only the $\mathrm{D}^{0}$ are used, to treat in the same way decays involving a $\mathrm{D}^{*+}$ and decays involving a $\mathrm{D}^{* 0}$. Due to the very good mass resolution, the three peaks corresponding to decays $\mathrm{B} \rightarrow \mathrm{D}^{*} \overline{\mathrm{D}}^{*} \mathrm{~K}, \mathrm{~B} \rightarrow \mathrm{D}^{*} \mathrm{~K}+\mathrm{D}^{*} \overline{\mathrm{D}} \mathrm{K}$ 


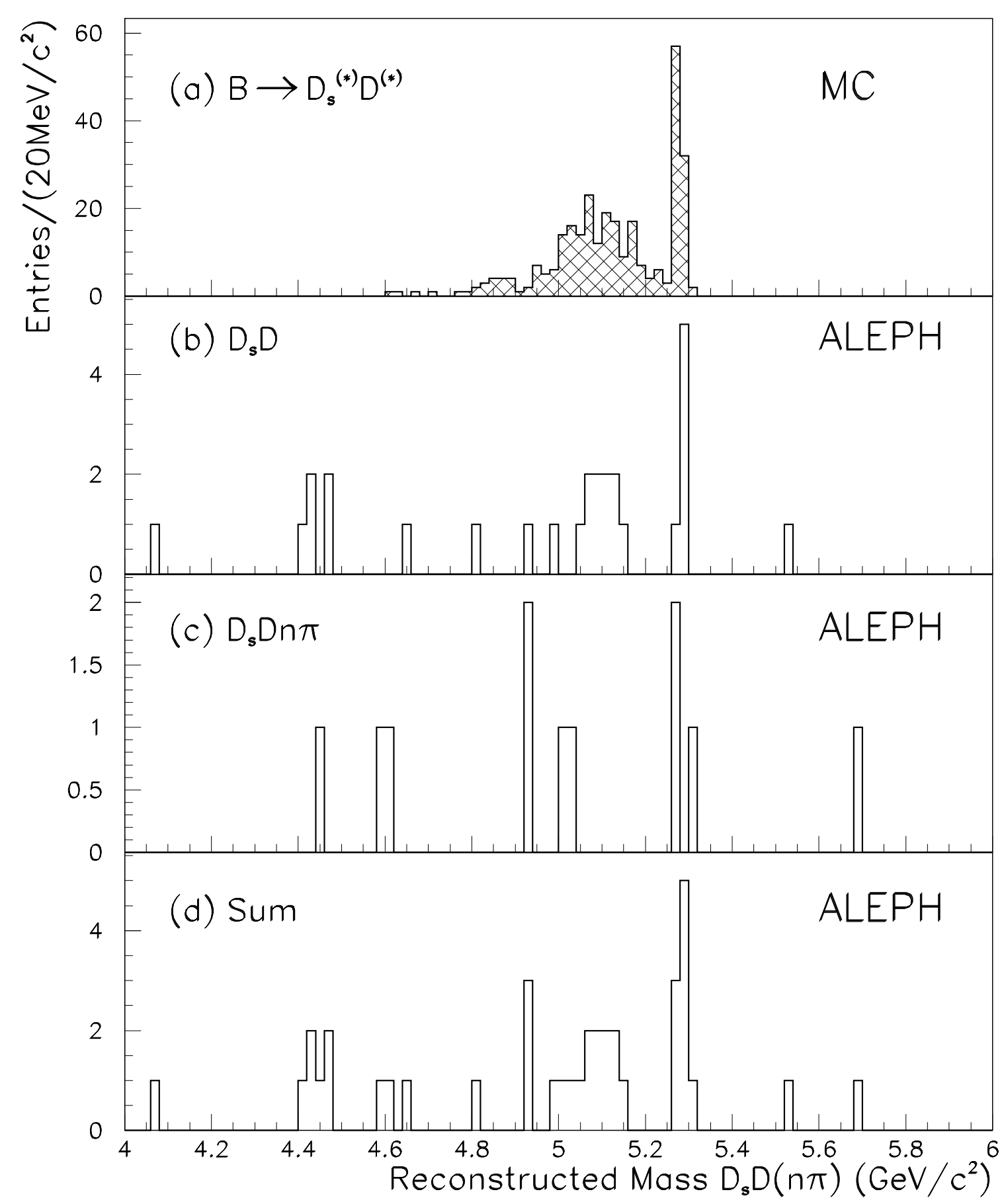

Figure 6: Invariant mass $m\left(\mathrm{D}_{\mathrm{s}}^{+} \overline{\mathrm{D}}\left(n \pi^{ \pm}\right)\right)$reconstructed for (a) Monte Carlo two-body decays $\mathrm{B} \rightarrow \mathrm{D}_{\mathrm{s}}^{(*)+} \mathrm{D}^{(*)-}$, and for ALEPH data (b) $\mathrm{D}_{\mathrm{s}}^{+} \overline{\mathrm{D}}$ (c) $\mathrm{D}_{\mathrm{s}}^{+} \overline{\mathrm{D}} n \pi^{ \pm}, n \geq 1$ (d) sum of all channels. The peak close to $5.1 \mathrm{GeV} / c^{2}$ is due to events with one missing neutral from decays $\mathrm{D}^{*} \rightarrow \mathrm{D} \pi^{0}, \gamma$ or or $\mathrm{D}_{\mathrm{s}}^{*+} \rightarrow \mathrm{D}_{\mathrm{s}}^{+} \gamma$. Here, $\overline{\mathrm{D}}$ is a generic term and can be either a reconstructed $\overline{\mathrm{D}}^{0}, \mathrm{D}^{-}$or $\mathrm{D}^{*-}$. 


\begin{tabular}{|c|c|c|c|}
\hline & N events & Comb. bkg. & Excess \\
\hline No tag & 256 & $180.3 \pm 10.2$ & $75.7 \pm 19.0$ \\
\hline $\mathrm{K}^{0}$ tag & 25 & $9.8 \pm 2.1$ & $15.2 \pm 5.4$ \\
$\mathrm{~K}^{ \pm}$tag & 29 & $11.6 \pm 2.5$ & $17.4 \pm 5.9$ \\
$\mathrm{~K}^{0}$ or K ${ }^{ \pm}$tag & 52 & $19.8 \pm 3.3$ & $32.2 \pm 7.9$ \\
\hline $\mathrm{K}^{0}$ tag (three-body) & 14 & $3.1 \pm 1.3$ & $10.9 \pm 3.9$ \\
$\mathrm{~K}^{ \pm}$tag (three-body) & 13 & $3.0 \pm 1.2$ & $10.0 \pm 3.8$ \\
$\mathrm{~K}^{0}$ or $\mathrm{K}^{ \pm}$tag (three-body) & 27 & $5.8 \pm 1.7$ & $21.2 \pm 5.5$ \\
\hline
\end{tabular}

Table 5. The observed signal and background in the $\mathrm{D} \overline{\mathrm{D}} X$ channel with and without associated kaon tag.

and $\mathrm{B} \rightarrow \mathrm{D} \overline{\mathrm{DK}}$ are clearly separated and can be fitted by 3 gaussians at average masses of 4.95, 5.11 and $5.28 \mathrm{GeV} / c^{2}$, and widths $(\sigma)$ of 32,21 and $11 \mathrm{MeV} / c^{2}$, respectively. Evidence for these peaks are also seen in the data, and the excess of events observed in the three-body mass window $4.80<\mathrm{m}(\mathrm{D} \overline{\mathrm{DK}})<5.32 \mathrm{GeV} / c^{2}$ indicates that a large part of the observed signal is indeed compatible with three-body decays $\mathrm{B} \rightarrow \mathrm{D}^{(*)} \overline{\mathrm{D}}^{(*)} \mathrm{K}$. These decays are studied more quantitatively in the following section.

\subsubsection{Analysis of three-body decays $B \rightarrow \mathrm{D}^{(*)} \overline{\mathrm{D}}^{(*)} \mathrm{K}$}

Apart from their experimental simplicity, three-body decays are interesting because they can probe the different quark amplitudes responsible for those decays. Moreover, it is possible to identify which $\mathrm{D}^{(*)}$ is from the $\mathrm{b}$ quark and which $\mathrm{D}^{(*)}$ is from the virtual $\mathrm{W}$ decay. The three-body decays may also include the resonant two-body decays $\mathrm{B} \rightarrow \overline{\mathrm{D}}^{(*)} \mathrm{D}_{\mathrm{s}}^{* *+}$ followed by $\mathrm{D}_{\mathrm{s}}^{* *+} \rightarrow \mathrm{D}^{(*)} \mathrm{K}$. Genuine three-body decays can proceed either through the external spectator diagram of Fig.5c or through the internal spectator diagram of Fig.5d. The decays $\mathrm{B}^{-} \rightarrow \mathrm{D}^{(*) 0} \mathrm{D}^{(*)} \mathrm{K}^{0}$ and $\overline{\mathrm{B}}^{0} \rightarrow \mathrm{D}^{(*)+} \overline{\mathrm{D}}^{(*) 0} \mathrm{~K}^{-}$can only occur through an external spectator amplitude (E). The decays $\mathrm{B}^{-} \rightarrow \mathrm{D}^{(*) 0} \overline{\mathrm{D}}^{(*) 0} \mathrm{~K}^{-}$and $\overline{\mathrm{B}}^{0} \rightarrow \mathrm{D}^{(*)+} \mathrm{D}^{(*)}-\mathrm{K}^{0}$ occur through the interference of both amplitudes (EI). The decays $\overline{\mathrm{B}}^{0} \rightarrow \mathrm{D}^{(*) 0} \overline{\mathrm{D}}^{(*) 0} \mathrm{~K}^{0}$ and $\mathrm{B}^{-} \rightarrow \mathrm{D}^{(*)+} \mathrm{D}^{(*)-} \mathrm{K}^{-}$ can occur only through an internal spectator amplitude (I): they are expected to be coloursuppressed and the measurement of their branching fraction would test the effectiveness of the colour suppression mechanism in B decays. To date, colour-suppressed B decays have only been seen through the occurence of decays $\mathrm{B} \rightarrow \psi, \chi_{\mathrm{c}} X$.

Three-body decays are searched for among the $\operatorname{DD} K(X)$ events selected in the previous section, by requiring that no additional charged track, incompatible with the interaction point, originates from the $\mathrm{D} \overline{\mathrm{DK}}$ vertex. The mass spectrum of the selected events is shown in Fig.8a $\left(D \bar{D} K^{0}\right)$ and Fig.8b $\left(D \bar{D} K^{ \pm}\right)$. Here, contrary to Fig.7, the $\pi^{ \pm}$from $D^{* \pm} \rightarrow D^{0} \pi^{ \pm}$have been included in the mass computation and $\mathrm{D}$ means therefore either a $\mathrm{D}^{0}$, a $\mathrm{D}^{+}$or a $\mathrm{D}^{*+}$. 

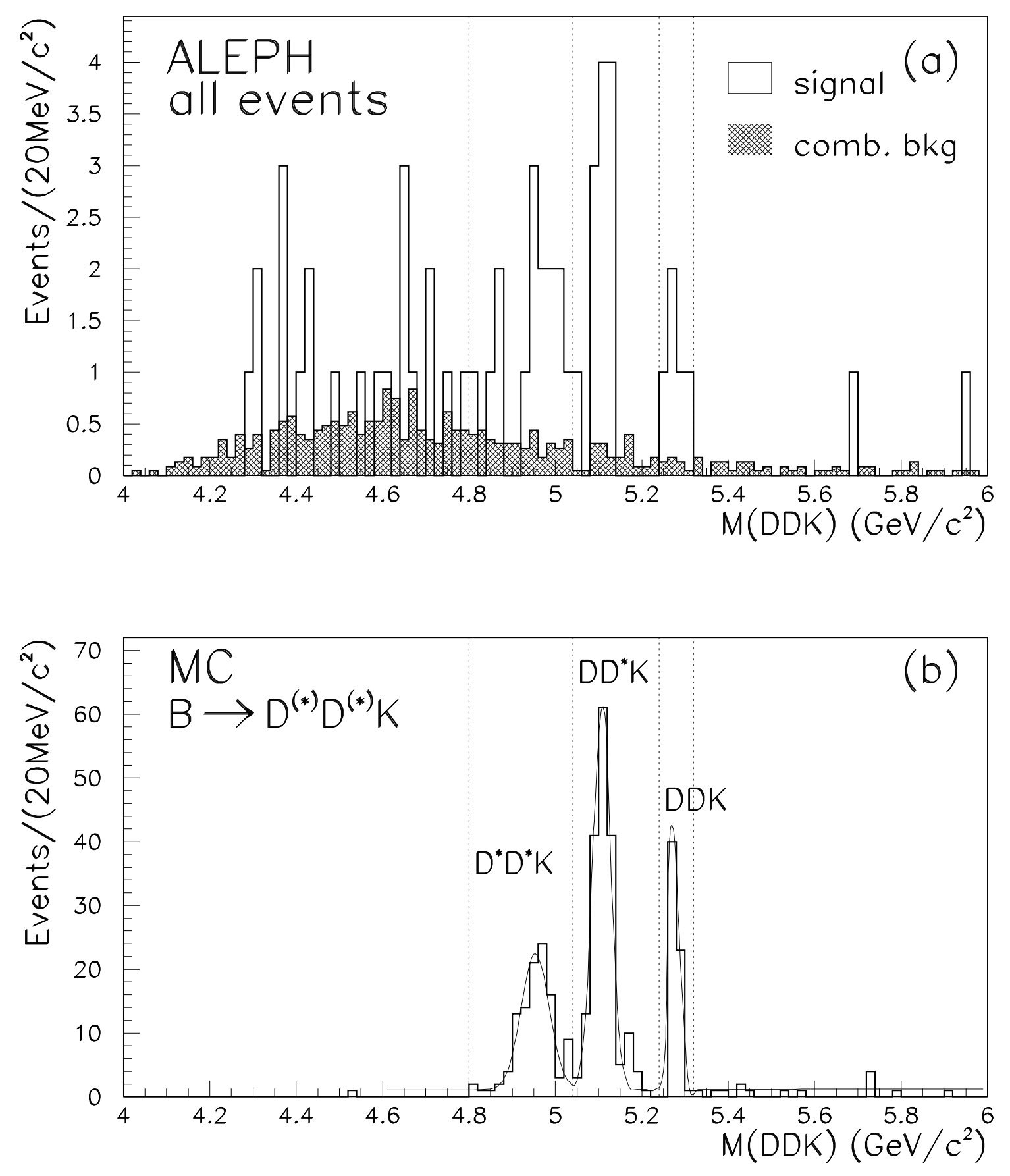

Figure 7: The $\mathrm{D}^{0} \overline{\mathrm{D}}^{0} \mathrm{~K}, \mathrm{D}^{0} \mathrm{D}^{-} \mathrm{K}$ or $\mathrm{D}^{+} \mathrm{D}^{-} \mathrm{K}$ mass of $\mathrm{D} \overline{\mathrm{D}}$ events with a reconstructed $\mathrm{K}_{\mathrm{S}}^{0}$ or a $\mathrm{K}^{ \pm}$for (a) ALEPH data (b) simulated three-body decays $\mathrm{B} \rightarrow \mathrm{D}^{(*)} \overline{\mathrm{D}}^{(*)} \mathrm{K}$. The $\pi^{+}$from $\mathrm{D}^{*+} \rightarrow \mathrm{D}^{0} \pi^{+}$, even if reconstructed, are not used in the mass. For the data, the distribution expected for combinatorial background events is also shown (shaded histogram). Its shape is obtained from the sideband events in the $D_{1}$ vs $D_{2}$ mass distributions, and its normalization is computed as explained in Section 5.1. 
Depending whether there are zero, one or two reconstructed $\mathrm{D}^{* \pm}$, the mass window for events compatible with a three-body $\mathrm{B} \rightarrow \mathrm{D}^{(*)} \overline{\mathrm{D}}^{(*)} \mathrm{K}$ decay is defined as $m_{0}<m(\mathrm{DD} \overline{\mathrm{D}})<5.32 \mathrm{GeV} / c^{2}$, with $m_{0}=4.80,5.04$ or $5.24 \mathrm{GeV} / c^{2}$ respectively. The number of signal and combinatorial background events found in the three-body DD̄K mass window is estimated with the technique used previously. The results are given in Table 5 and show that a large fraction of the signal events are indeed compatible with a three-body $\mathrm{B} \rightarrow \mathrm{D}^{(*)} \overline{\mathrm{D}}^{(*)} \mathrm{K}$ hypothesis. However, some events in the lowest mass peak region of Fig.7 and Fig.8 $\left(4.80<m(\mathrm{DDK})<5.04 \mathrm{GeV} / c^{2}\right)$ are also compatible with a four-body $\mathrm{B} \rightarrow \mathrm{D} \overline{\mathrm{DK}} \pi$ decay hypothesis where the $\pi$ has not been seen. Because of this ambiguity, they are not used to extract the three-body decay branching fractions in the following.

Seven candidates for completely reconstructed decays $\mathrm{B} \rightarrow \mathrm{D}^{(*)} \overline{\mathrm{D}}^{(*)} \mathrm{K}_{\mathrm{S}}^{0}$ and five candidates for completely reconstructed decays $\mathrm{B} \rightarrow \mathrm{D}^{(*)} \overline{\mathrm{D}}^{(*)} \mathrm{K}^{ \pm}$are obtained over a combinatorial background of $0.4 \pm 0.1$ and $0.3 \pm 0.1$ events respectively. Two candidates for partially reconstructed $\mathrm{B} \rightarrow \mathrm{D}^{(*)} \overline{\mathrm{D}}^{(*)} \mathrm{K}_{\mathrm{S}}^{0}$ (background $1.0 \pm 0.3$ events) and four candidates for partially reconstructed $\mathrm{B} \rightarrow \mathrm{D}^{(*)} \overline{\mathrm{D}}^{(*)} \mathrm{K}^{ \pm}$(background $1.0 \pm 0.3$ events) are also observed at $5.04<$ $m(\mathrm{D} \overline{\mathrm{DK}})<5.24 \mathrm{GeV} / c^{2}$ : these events are compatible with three-body decays where a $\pi^{0}$ or $\gamma$ from $\mathrm{D}^{*} \rightarrow \mathrm{D} \pi^{0}, \gamma$ was missed. The branching fractions for the different possible three-body decays $\mathrm{B} \rightarrow \mathrm{D}^{(*)} \overline{\mathrm{D}}^{(*)} \mathrm{K}$ are obtained from the number of events observed in each channel at $5.24<m(\mathrm{D} \overline{\mathrm{DK}})<5.32 \mathrm{GeV} / c^{2}$ (completely reconstructed decays) and at $5.04<m(\mathrm{D} \overline{\mathrm{DK}})<$ $5.24 \mathrm{GeV} / c^{2}$ (events involving one unreconstructed $\mathrm{D}^{* 0} \rightarrow \mathrm{D}^{0} \pi^{0}, \gamma$ or $\mathrm{D}^{*+} \rightarrow \mathrm{D}^{+} \pi^{0}$ ). The selection efficiencies are computed using a sample of Monte Carlo events involving three-body decays $\mathrm{B} \rightarrow \mathrm{D}^{(*)} \overline{\mathrm{D}}^{(*)} \mathrm{K}$. The branching fractions $\mathcal{B}\left(\mathrm{B} \rightarrow \mathrm{D}_{1} \mathrm{D}_{2} \mathrm{~K}\right)$ are extracted by maximizing the likelihood from Equation (2), where $n_{i, j}$ is now given by

$$
\bar{n}_{i j}=N_{\mathrm{bkg}}(i, j)+2 N(\mathrm{Z}) \frac{\Gamma_{\mathrm{b} \overline{\mathrm{b}}}}{\Gamma_{\mathrm{had}}} \mathrm{f}_{\mathrm{B}_{\mathrm{d}}^{0}} \mathcal{B}\left(\mathrm{B} \rightarrow \mathrm{D}_{1} \mathrm{D}_{2} \mathrm{~K}\right) \mathcal{B}\left(\mathrm{D}_{1} \rightarrow i\right) \mathcal{B}\left(\mathrm{D}_{2} \rightarrow j\right) \epsilon_{i j}
$$

Here, the efficiency $\epsilon_{i j}$ incorporates also the $\mathrm{K}$ reconstruction efficiency. The sum over $i, j$ is performed over all possible contributing channels (for instance, a decay $\mathrm{B} \rightarrow \mathrm{D}^{0} \mathrm{D}^{*+} \mathrm{K}$ can be detected either in the channel $\mathrm{D}^{0} \mathrm{D}^{*+} \mathrm{K}$ with $5.24<m\left(\mathrm{D}^{0} \mathrm{D}^{*+} \mathrm{K}\right)<5.32 \mathrm{GeV} / c^{2}$ or in the channel $\mathrm{D}^{0} \mathrm{D}^{+} \mathrm{K}$ with $\left.5.04<m\left(\mathrm{D}^{0} \mathrm{D}^{+} \mathrm{K}\right)<5.24 \mathrm{GeV} / c^{2}\right)$. The sharing of the background between the individual channels is assumed to be the same as in the inclusive analysis.

In order to increase the statistics per channel, the isospin symmetry of these decays is used $[18,19]$, and the $\mathrm{B}^{0}$ and $\mathrm{B}^{+}$branching fractions corresponding to the same decay amplitude are assumed equal. The average B branching fractions found for each decay amplitude are summarized in Table 6. For the channels with no detected signal or a low significance, a $90 \%$ C.L. upper limit on the branching fraction is extracted. For the other channels, the first error on $\mathcal{B}$ is statistical, the second one is the systematic resulting from Monte Carlo statistics, detector simulation, uncertainty on the combinatorial background and uncertainty on $\mathrm{f}_{\mathrm{B}_{\mathrm{d}}^{0}}$, and the last one is the error resulting from the uncertainty on the different $\mathrm{D}$ branching fractions. For decays $\mathrm{B} \rightarrow \mathrm{D}^{*} \overline{\mathrm{D}}^{*} \mathrm{~K}$ corresponding to I or EI transitions, only the results from $\mathrm{B} \rightarrow \mathrm{D}^{*+} \overline{\mathrm{D}}^{*-} \mathrm{K}$ are used 

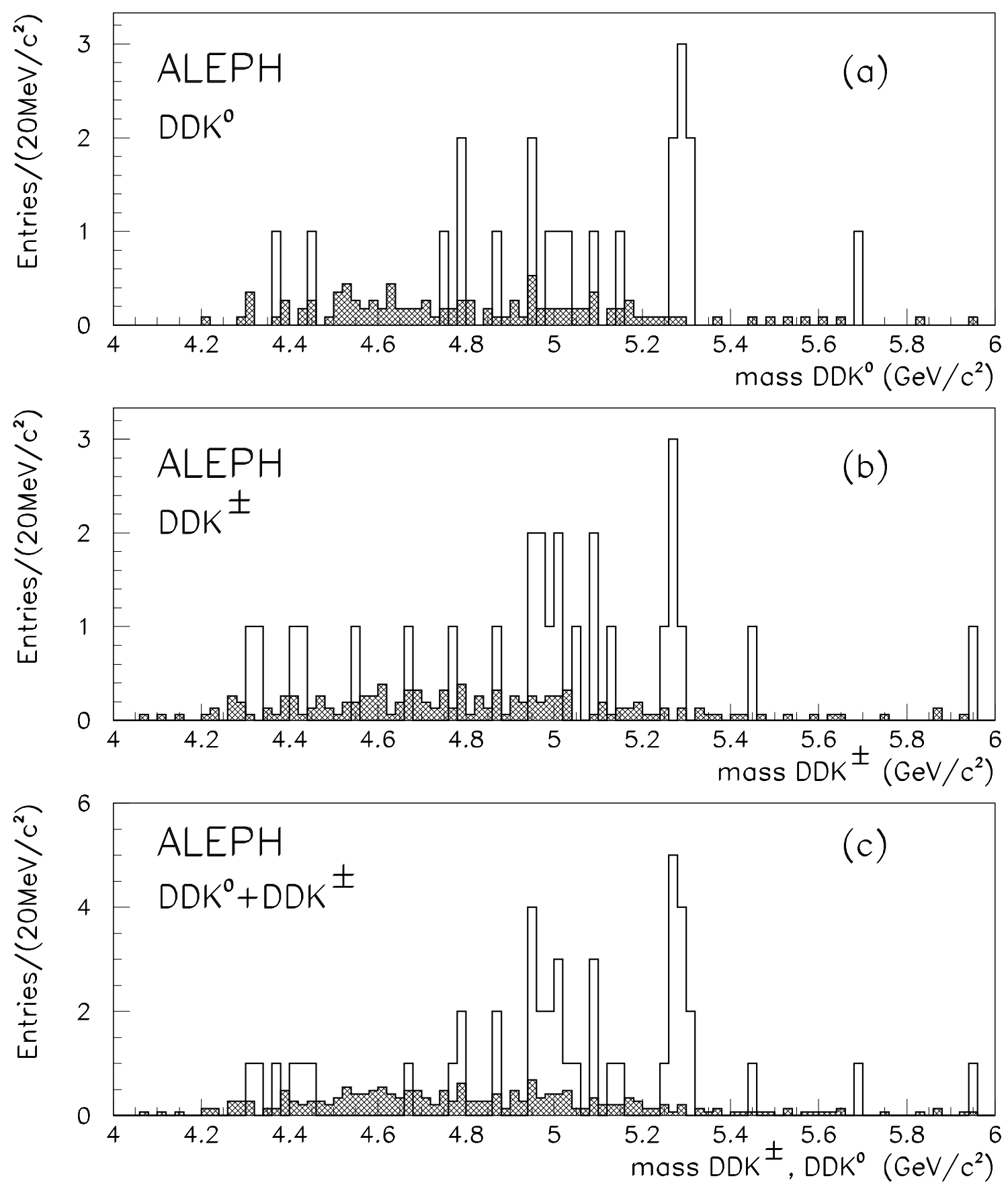

Figure 8: Invariant mass $m(\mathrm{D} \overline{\mathrm{DK}})$ for events with one identified $\mathrm{K}$ and no other additional track from the DD̄K vertex. D can be either a $\mathrm{D}^{0}$, a $\mathrm{D}^{+}$or a $\mathrm{D}^{*+}$. (a) Events $\mathrm{D} \overline{\mathrm{D}} \mathrm{K}^{0}$, (b) events $\mathrm{DD}^{ \pm}$, (c) sum of both channels. The distribution expected for combinatorial background events is also shown (shaded histogram). Its shape is obtained from the sideband events in the $\mathrm{D}_{1}$ vs $\mathrm{D}_{2}$ mass distributions, and its normalization is computed as explained in Section 5.1. 


\begin{tabular}{|c|c|c|c|}
\hline Diagram & $\begin{array}{l}\text { Channel } \\
\left(\mathrm{B}^{0}, \mathrm{~B}^{+}\right)\end{array}$ & $\begin{array}{l}\text { Number of } \\
\text { candidates }\end{array}$ & $\begin{array}{c}\mathcal{B}\left(\mathrm{B} \rightarrow \mathrm{D}^{(*)} \overline{\mathrm{D}}^{(*)} \mathrm{K}\right) \\
\left(\mathrm{B}^{0} / \mathrm{B}^{+} \text {average }\right)\end{array}$ \\
\hline $\mathrm{E}$ & $\mathrm{D}^{-} \mathrm{D}^{0} \mathrm{~K}^{+}, \overline{\mathrm{D}}^{0} \mathrm{D}^{+} \mathrm{K}^{0}$ & 3 & $1.7_{-0.8}^{+1.2} \pm 0.2 \pm 0.1 \%$ \\
\hline $\mathrm{E}$ & $\left(\mathrm{D}^{*-} \mathrm{D}^{0}+\mathrm{D}^{-} \mathrm{D}^{* 0}\right) \mathrm{K}^{+},\left(\overline{\mathrm{D}}^{* 0} \mathrm{D}^{+}+\overline{\mathrm{D}}^{0} \mathrm{D}^{*+}\right) \mathrm{K}^{0}$ & 5 & $1.8_{-0.8}^{+1.0} \pm 0.3 \pm 0.1 \%$ \\
\hline $\mathrm{E}$ & $\mathrm{D}^{*-} \mathrm{D}^{* 0} \mathrm{~K}^{+}, \overline{\mathrm{D}}^{* 0} \mathrm{D}^{*+} \mathrm{K}^{0}$ & 1 & $<1.3 \%$ \\
\hline $\mathrm{I}$ & $\overline{\mathrm{D}}^{0} \mathrm{D}^{0} \mathrm{~K}^{0}, \mathrm{D}^{+} \mathrm{D}^{-} \mathrm{K}^{+}$ & 1 & $<2.0 \%$ \\
\hline I & $\left(\overline{\mathrm{D}}^{0} \mathrm{D}^{* 0}+\overline{\mathrm{D}}^{* 0} \mathrm{D}^{0}\right) \mathrm{K}^{0},\left(\mathrm{D}^{*+} \mathrm{D}^{-}+\mathrm{D}^{+} \mathrm{D}^{*-}\right) \mathrm{K}^{+}$ & 1 & $<1.6 \%$ \\
\hline $\mathrm{I}$ & $\overline{\mathrm{D}}^{* 0} \mathrm{D}^{* 0} \mathrm{~K}^{0}, \mathrm{D}^{*+} \mathrm{D}^{*-} \mathrm{K}^{+}$ & 1 & $<1.5 \%$ \\
\hline EI & $\mathrm{D}^{+} \mathrm{D}^{-} \mathrm{K}^{0}, \overline{\mathrm{D}}^{0} \mathrm{D}^{0} \mathrm{~K}^{+}$ & 1 & $<1.9 \%$ \\
\hline EI & $\left(\mathrm{D}^{*+} \mathrm{D}^{-}+\mathrm{D}^{+} \mathrm{D}^{*-}\right) \mathrm{K}^{0},\left(\overline{\mathrm{D}}^{* 0} \mathrm{D}^{0}+\overline{\mathrm{D}}^{0} \mathrm{D}^{* 0}\right) \mathrm{K}^{+}$ & 4 & $1.6_{-0.7}^{+1.0} \pm 0.2 \pm 0.1 \%$ \\
\hline EI & $\mathrm{D}^{*+} \mathrm{D}^{*-} \mathrm{K}^{0}, \overline{\mathrm{D}}^{* 0} \mathrm{D}^{* 0} \mathrm{~K}^{+}$ & 1 & $<3.0 \%$ \\
\hline Sum E & 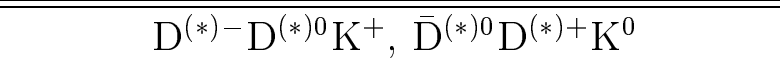 & 9 & $3.5_{-1.1}^{+1.7}{ }_{-0.4}^{+0.5} \pm 0.2 \%$ \\
\hline Sum I & $\overline{\mathrm{D}}^{(*) 0} \mathrm{D}^{(*) 0} \mathrm{~K}^{0}, \mathrm{D}^{(*)+} \mathrm{D}^{(*)-} \mathrm{K}^{+}$ & 3 & $0.8_{-0.4}^{+1.0}{ }_{-0.1}^{+0.2} \pm 0.1 \%$ \\
\hline Sum EI & $\mathrm{D}^{(*)+} \mathrm{D}^{(*)-} \mathrm{K}^{0}, \overline{\mathrm{D}}^{(*) 0} \mathrm{D}^{(*) 0} \mathrm{~K}^{+}$ & 6 & $2.8_{-1.0}^{+1.6}{ }_{-0.3}^{+0.4} \pm 0.2 \%$ \\
\hline $\mathrm{E}+\mathrm{I}+\mathrm{EI}$ & Sum DD̄K & 5 & $2.3_{-0.9}^{+1.5}{ }_{-0.3}^{+0.3} \pm 0.2 \%$ \\
\hline $\mathrm{E}+\mathrm{I}+\mathrm{EI}$ & Sum $\mathrm{D}^{*} \mathrm{~K}+\mathrm{D}^{*} \overline{\mathrm{D}} \mathrm{K}$ & 10 & $3.8_{-1.1}^{+1.6}{ }_{-0.4}^{+0.5} \pm 0.2 \%$ \\
\hline $\mathrm{E}+\mathrm{I}+\mathrm{EI}$ & Sum $D^{*} \bar{D}^{*} \mathrm{~K}$ & 3 & $1.0_{-0.6}^{+1.3}{ }_{-0.2}^{+0.2} \pm 0.1 \%$ \\
\hline $\mathrm{E}+\mathrm{I}+\mathrm{EI}$ & Sum $\mathrm{D}^{(*)} \overline{\mathrm{D}}^{(*)} \mathrm{K}$ & 18 & $7.1_{-1.5}^{+2.5}{ }_{-0.8}^{+0.9} \pm 0.5 \%$ \\
\hline
\end{tabular}

Table 6. Summary of the various branching fractions $B \rightarrow$ DDK measured in this analysis. For the channels with no significant signal, the upper limits are given for a $90 \%$ confidence level.

in the $\mathrm{B}$ average, since no $\mathrm{B} \rightarrow \overline{\mathrm{D}}^{* 0} \mathrm{D}^{* 0} \mathrm{~K}$ measurement is performed. The largest branching fractions are measured for decays possible through an external spectator amplitude (E or EI). The total branching fraction for three-body decays is

$$
\mathcal{B}\left(\mathrm{B} \rightarrow \mathrm{D}^{(*)} \overline{\mathrm{D}}^{(*)} \mathrm{K}\right)=\left(7.1_{-1.5}^{+2.5}{ }_{-0.8}^{+0.9} \pm 0.5\right) \%
$$

Compared to the result of Table 3

$$
\mathcal{B}\left(\mathrm{b} \rightarrow \mathrm{D}^{0} \overline{\mathrm{D}}^{0}, \mathrm{D}^{0} \mathrm{D}^{-}, \mathrm{D}^{+} \overline{\mathrm{D}}^{0}(X)\right)=\left(7.8_{-1.8}^{+2.0}{ }_{-1.5}^{+1.7}{ }_{-0.4}^{+0.5}\right) \%
$$

scaled by a factor $1 / 2 \mathrm{f}_{\mathrm{B}_{\mathrm{d}}^{0}}=1.3$ to account for $\mathrm{b} \rightarrow \mathrm{B}^{0}, \mathrm{~B}^{ \pm}$, one sees that the three-body decays $\mathrm{B} \rightarrow \overline{\mathrm{D}}^{(*)} \mathrm{D}^{(*)} \mathrm{K}$ are a large part (about $70 \%$ ) of the inclusive $\mathrm{B} \rightarrow \mathrm{D} \overline{\mathrm{D}}(X)$ decays.

The event properties for the eighteen three-body decay candidates discussed above are given in Appendix 1, Tables 11 and 12. From the invariant mass of the allowed DK combinations, no evidence for decays $\mathrm{B} \rightarrow \overline{\mathrm{D}}^{(*)} \mathrm{D}_{\mathrm{s} 1}^{+}$followed by $\mathrm{D}_{\mathrm{s} 1}^{+} \rightarrow \mathrm{D}^{*} \mathrm{~K}$ is found: the $\mathrm{D}_{\mathrm{s} 1}^{+}$should appear at a mass of $2535 \mathrm{MeV} / c^{2}$ in $\mathrm{D}^{*+} \mathrm{K}^{0}$ (completely reconstructed decays) and about $2390 \mathrm{MeV} / c^{2}$ in $\mathrm{D}^{0} \mathrm{~K}^{+}$or $\mathrm{D}^{+} \mathrm{K}^{0}$ (partially reconstructed decays with one unreconstructed neutral from $\left.\mathrm{D}^{*} \rightarrow \mathrm{D} \pi^{0}, \gamma\right)$. No resonant substructure in the $\overline{\mathrm{DD}}$ mass of the selected candidates is found 
either. For the thirteen events where the $\mathrm{D}$ from $\mathrm{b}\left(\mathrm{D}_{1}\right)$ can be distinguished from the $\mathrm{D}$ from $\mathrm{W}\left(\mathrm{D}_{2}\right)$, the invariant mass $m\left(\mathrm{D}_{1} \mathrm{~K}\right)$ tend to be higher than $m\left(\mathrm{D}_{2} \mathrm{~K}\right)$ (and hence the momentum $p\left(\mathrm{D}_{1}\right)$ in the $\mathrm{B}$ rest frame is higher than $\left.p\left(\mathrm{D}_{2}\right)\right)$. However, after the Lorentz boost, the distributions of $p\left(\mathrm{D}_{1}\right)$ and $p\left(\mathrm{D}_{2}\right)$ in the laboratory are quite similar.

\subsection{Search for Cabibbo suppressed decays $B \rightarrow \bar{D}^{(*)} \mathrm{D}^{(*)+}$}

The decay $\overline{\mathrm{b}} \rightarrow \overline{\mathrm{c}} \mathrm{W}^{+}$followed by the Cabibbo suppressed amplitude $\mathrm{W}^{+} \rightarrow \mathrm{c} \overline{\mathrm{d}}$ (Fig.5e) can give a small contribution to the observed $\overline{\mathrm{D}} \mathrm{D}$ signal in the mass region $m(\overline{\mathrm{D}} \mathrm{D}) \simeq m(\mathrm{~B})$. The two-body decays $\mathrm{B} \rightarrow \overline{\mathrm{D}}^{(*)} \mathrm{D}^{(*)+}$ are expected to be suppressed by a factor $\tan \theta_{C}{ }^{2} \simeq 1 / 20$ relative to the two-body decays $\mathrm{B} \rightarrow \overline{\mathrm{D}}^{(*)} \mathrm{D}_{\mathrm{s}}^{(*)+}$, leading to an expected branching fraction $\mathcal{B}\left(\mathrm{B} \rightarrow \overline{\mathrm{D}}^{(*)} \mathrm{D}^{(*)+}\right) \simeq 0.3 \%$ if one uses the values measured in Section 6.2. The two-body decays $\mathrm{B}^{0} \rightarrow \mathrm{D}^{(*)-} \mathrm{D}^{(*)+}$ are especially interesting since they are favorable modes for testing $\mathrm{CP}$ violation in $\mathrm{B}$ decays at future $\mathrm{B}$ factories. These decays have never been observed previously.

From the $\bar{D} D$ mass distribution of the events selected in the inclusive analysis (Fig.4), two candidates for completely reconstructed decays $\mathrm{B}^{0} \rightarrow \mathrm{D}^{*-} \mathrm{D}^{*+}$ and four candidates for partially reconstructed decays $\mathrm{B}^{+} \rightarrow \overline{\mathrm{D}}^{(*) 0} \mathrm{D}^{(*)+}$ with a $\overline{\mathrm{D}}^{0} \mathrm{D}^{+}$pair in the final state are observed. The combinatorial background in the signal region is estimated by fitting the background distributions shown in Fig.4 to an exponential or a second order polynomial. The results are summarized in Table 7. The numbers of events expected in each channel for a branching fraction of $0.1 \%$ are also indicated in the table, as well as the D $\bar{D}$ mass window used to search for a signal. From these results, a $90 \%$ confidence level upper limit on the individual $\mathrm{B}^{0}, \mathrm{~B}^{+}$ and on the average $\mathrm{B}$ branching fractions is derived (Table 8). The results for the average of $\mathrm{B}^{0}$ and $\mathrm{B}^{+}$decays is computed assuming equality of the corresponding $\mathrm{B}^{0}$ and $\mathrm{B}^{+}$branching fractions.

The parameters of the six candidates are given in Table 9. The more significant channel is $\mathrm{B}^{0} \rightarrow \mathrm{D}^{*-} \mathrm{D}^{*+}$, where two candidates are observed over a combinatorial background of $0.10 \pm 0.03$ events. Assuming the two candidates are signal, the corresponding branching fraction is:

$$
\mathcal{B}\left(\mathrm{B}^{0} \rightarrow \mathrm{D}^{*+} \mathrm{D}^{*-}\right)=\left(0.23_{-0.12}^{+0.19} \pm 0.04 \pm 0.02\right) \% .
$$

The first error on $\mathcal{B}$ is statistical, the second one is the systematic resulting from Monte Carlo statistics, detector simulation and uncertainty on $\mathrm{f}_{\mathrm{B}_{\mathrm{d}}^{0}}$, and the last one is from the uncertainty on the different $\mathrm{D}$ branching fractions. However, taking into account the uncertainty on the combinatorial background, the probability that the two $\mathrm{D}^{*+} \mathrm{D}^{*-}$ candidates result from a statistical fluctuation of the background is still at the $1 \%$ level. Therefore, their compatibility with the $\mathrm{B}^{0} \rightarrow \mathrm{D}^{*-} \mathrm{D}^{*+}$ decay hypothesis is now examined. The selection is tightened, discarding events with additional tracks at the $\mathrm{B}$ vertex that are incompatible with the interaction point, or additional $\mathrm{K}_{\mathrm{S}}^{0}$ in the $\mathrm{D} \overline{\mathrm{D}}$ hemisphere. A cut $x_{E}>0.70$, where $x_{E}=E_{\mathrm{D} \overline{\mathrm{D}}} / E_{\text {beam }}$, is also applied: because of the hard B fragmentation, most fully reconstructed 


\begin{tabular}{|c|c|c|c|c|c|}
\hline $\begin{array}{c}\text { Decay } \\
\text { channel }\end{array}$ & $\begin{array}{c}\text { Detection } \\
\text { channel }\end{array}$ & $\begin{array}{c}\text { Predicted \# } \\
\text { of events } \\
(\mathcal{B}=0.1 \%)\end{array}$ & $\begin{array}{c}\text { Signal } \\
\text { mass window } \\
\left(\mathrm{GeV} / c^{2}\right)\end{array}$ & $\begin{array}{c}\text { Events } \\
\text { seen }\end{array}$ & $\begin{array}{c}\text { Comb. } \\
\text { bkg. }\end{array}$ \\
\hline$\overline{\mathrm{B}}^{0} \rightarrow$ & $\mathrm{D}^{*+} \mathrm{D}^{*-}$ & 0.56 & $5.24-5.32$ & 2 & $0.10 \pm 0.03$ \\
$\mathrm{D}^{*+} \mathrm{D}^{*-}$ & $\mathrm{D}^{-} \mathrm{D}^{*+}, \mathrm{D}^{+} \mathrm{D}^{*-}$ & 0.27 & $5.04-5.24$ & 0 & $0.47 \pm 0.15$ \\
& $\mathrm{D}^{-} \mathrm{D}^{+}$ & 0.04 & $4.80-5.04$ & 0 & $0.79 \pm 0.12$ \\
$\mathrm{D}^{+} \mathrm{D}^{*-}+\mathrm{D}^{*+} \mathrm{D}^{-}$ & $\mathrm{D}^{-} \mathrm{D}^{*+}, \mathrm{D}^{+} \mathrm{D}^{*-}$ & 0.43 & $5.24-5.32$ & 0 & $0.11 \pm 0.04$ \\
& $\mathrm{D}^{-} \mathrm{D}^{+}$ & 0.12 & $5.04-5.24$ & 0 & $0.44 \pm 0.09$ \\
$\mathrm{D}^{+} \mathrm{D}^{-}$ & $\mathrm{D}^{+} \mathrm{D}^{-}$ & 0.39 & $5.24-5.32$ & 0 & $0.12 \pm 0.05$ \\
\hline $\mathrm{B}^{+} \rightarrow$ & $\mathrm{D}^{0} \mathrm{D}^{*+}$ & 0.37 & $5.04-5.24$ & 0 & $0.78 \pm 0.10$ \\
$\mathrm{D}^{* 0} \mathrm{D}^{*+}$ & $\mathrm{D}^{0} \mathrm{D}^{+}$ & 0.11 & $4.80-5.04$ & 2 & $1.41 \pm 0.25$ \\
& $\mathrm{D}^{0} \mathrm{D}^{*+}$ & 0.19 & $5.24-5.32$ & 0 & $0.20 \pm 0.05$ \\
$\mathrm{D}^{0} \mathrm{D}^{*+}+\mathrm{D}^{* 0} \mathrm{D}^{+}$ & $\mathrm{D}^{0} \mathrm{D}^{+}$ & 0.22 & $5.04-5.24$ & 2 & $0.65 \pm 0.09$ \\
& $\mathrm{D}^{0} \mathrm{D}^{+}$ & 0.34 & $5.24-5.32$ & 0 & $0.16 \pm 0.03$ \\
\hline $\mathrm{D}^{0} \mathrm{D}^{+}$ & & &
\end{tabular}

Table 7. Detection channel, reconstructed $\mathrm{D}^{(*)} \mathrm{D}^{(*)}$ mass window for the signal events, expected and observed number of events in the signal region for the different two-body Cabibbo suppressed B decays. The expected number of events have been computed assuming a $0.1 \%$ branching fraction.

$\mathrm{B}$ mesons should have a large energy. When both cuts are applied to Monte Carlo $\mathrm{Z} \rightarrow \mathrm{q} \overline{\mathrm{q}}$ or $\mathrm{Z} \rightarrow \mathrm{b} \overline{\mathrm{b}}$ events, $64 \%$ of the $\mathrm{B} \rightarrow \mathrm{D} \overline{\mathrm{D}}$ decays reconstructed in the inclusive analysis are retained, for only $8 \%$ of the combinatorial background (over the whole $\mathrm{D} \overline{\mathrm{D}}$ mass region) and $31 \%$ of the combinatorial background at $m(\mathrm{DD})>4.8 \mathrm{GeV} / c^{2}$. The two $\mathrm{D}^{*+} \mathrm{D}^{*-}$ candidates survive the additional cuts.

Close scrutiny of the remaining $\overline{\mathrm{D}}^{0} \mathrm{D}^{+}$candidates listed in table 9 shows that they have some interesting properties, although no branching fraction measurements can be made. For instance, in event F (Fig.9) both D's are well separated from the DD vertex, and the latter is more than $3 \mathrm{~mm}$ away from the interaction point. Moreover, a $\pi^{0}$ of momentum $p\left(\pi^{0}\right)=2.9 \mathrm{GeV} / c$, compatible with the hypothesis $\mathrm{D}^{*-} \rightarrow \mathrm{D}^{-} \pi^{0}$, is reconstructed. The event is compatible with a decay $\mathrm{B}^{-} \rightarrow \mathrm{D}^{*-} \mathrm{D}^{0}$ and no other plausible explanation is found.

\subsection{Search for decays $\mathrm{B}^{0} \rightarrow \mathrm{D}^{(*) 0} \overline{\mathrm{D}}^{(*) 0}$}

The decays $\mathrm{B}^{0} \rightarrow \mathrm{D}^{(*) 0} \overline{\mathrm{D}}^{(*) 0}$ are forbidden in the spectator model: neither colour favoured, colour suppressed nor penguin amplitudes can lead to such final states. They can only occur through the W exchange diagram of Fig.5f. This leads to decay amplitudes suppressed by 


\begin{tabular}{|c|c|}
\hline Decay channel & 90\%C.L. Upper limit on $\mathcal{B}$ \\
\hline$\overline{\mathrm{B}}^{0} \rightarrow \mathrm{D}^{*+} \mathrm{D}^{*-}$ & $<0.61 \%$ \\
$\overline{\mathrm{B}}^{0} \rightarrow \mathrm{D}^{+} \mathrm{D}^{*-}+\mathrm{D}^{*+} \mathrm{D}^{-}$ & $<0.56 \%$ \\
$\overline{\mathrm{B}}^{0} \rightarrow \mathrm{D}^{-} \mathrm{D}^{+}$ & $<0.59 \%$ \\
\hline $\mathrm{B}^{+} \rightarrow \mathrm{D}^{* 0} \mathrm{D}^{*+}$ & $<1.11 \%$ \\
$\mathrm{~B}^{+} \rightarrow \mathrm{D}^{0} \mathrm{D}^{*+}+\mathrm{D}^{* 0} \mathrm{D}^{+}$ & $<1.30 \%$ \\
$\mathrm{~B}^{+} \rightarrow \mathrm{D}^{0} \mathrm{D}^{+}$ & $<0.67 \%$ \\
\hline average $\overline{\mathrm{B}}^{0}, \mathrm{~B}^{+}$ & $<0.59 \%$ \\
$\overline{\mathrm{B}} \rightarrow \mathrm{D}^{*} \mathrm{D}^{*-}$ & $<0.55 \%$ \\
$\overline{\mathrm{B}} \rightarrow \mathrm{DD} \mathrm{D}^{*-}+\mathrm{D}^{*} \mathrm{D}^{-}$ & $<0.31 \%$ \\
$\overline{\mathrm{B}} \rightarrow \mathrm{DD}^{-}$ &
\end{tabular}

Table 8. Branching fraction measurements for the two-body Cabibbo suppressed B decays.

\begin{tabular}{|c|c|c|c|c|c|c|}
\hline Event & $\mathrm{A}$ & $\mathrm{B}$ & $\mathrm{C}$ & $\mathrm{D}$ & $\mathrm{E}$ & $\mathrm{F}$ \\
\hline $\mathrm{D}_{1}$ & $\mathrm{D}^{*-}$ & $\mathrm{D}^{*-}$ & $\mathrm{D}^{-}$ & $\mathrm{D}^{+}$ & $\mathrm{D}^{+}$ & $\mathrm{D}^{-}$ \\
$\mathrm{D}_{1}$ decay mode & $\mathrm{K} \pi$ & $\mathrm{K} \pi \pi \pi$ & $\mathrm{K} \pi \pi$ & $\mathrm{K} \pi \pi$ & $\mathrm{K} \pi \pi$ & $\mathrm{K} \pi \pi$ \\
$\mathcal{P}\left(\mathrm{D}_{\mathrm{s}}^{+}\right)$ & - & - & $<10^{-10}$ & 0.33 & $<10^{-7}$ & $<10^{-7}$ \\
$\mathrm{D}_{2}$ & $\mathrm{D}^{*+}$ & $\mathrm{D}^{*+}$ & $\mathrm{D}^{0}$ & $\overline{\mathrm{D}}^{0}$ & $\overline{\mathrm{D}}^{0}$ & $\mathrm{D}^{0}$ \\
$\mathrm{D}_{2}$ decay mode & $\mathrm{K} \pi \pi^{0}$ & $\mathrm{~K} \pi$ & $\mathrm{K} \pi \pi \pi$ & $\mathrm{K} \pi \pi \pi$ & $\mathrm{K} \pi$ & $\mathrm{K} \pi \pi \pi$ \\
$x_{E}\left(\overline{\mathrm{D}}^{(*)} \mathrm{D}^{(*)}\right)$ & 0.80 & 0.81 & 0.79 & 0.60 & 0.80 & 0.81 \\
$p\left(\mathrm{D}_{1}\right)(\mathrm{GeV} / c)$ & 11.7 & 17.5 & 16.3 & 18.1 & 18.7 & 24.0 \\
$p\left(\mathrm{D}_{2}\right)(\mathrm{GeV} / c)$ & 24.6 & 19.4 & 18.6 & 9.0 & 17.6 & 11.8 \\
$m\left(\mathrm{D}_{1} \mathrm{D}_{2}\right)\left(\mathrm{GeV} / c^{2}\right)$ & 5.29 & 5.26 & 5.01 & 5.13 & 4.86 & 5.05 \\
$d_{\mathrm{B}(\mathrm{mm})}$ & $1.6 \pm 0.3$ & $0.3 \pm 0.2$ & $5.8 \pm 0.2$ & $4.0 \pm 0.2$ & $4.5 \pm 0.2$ & $3.2 \pm 0.2$ \\
$d_{\mathrm{BD}_{1}} / \sigma$ & +5.7 & +5.7 & +37.0 & +4.6 & +0.8 & +28.4 \\
$d_{\mathrm{BD}_{2}} / \sigma$ & +0.5 & +6.7 & +0.9 & +1.6 & +3.1 & +3.7 \\
\hline
\end{tabular}

Table 9. Properties of the 6 Cabibbo suppressed $\mathrm{B} \rightarrow \mathrm{D}^{(*)} \overline{\mathrm{D}}^{(*)}$ candidates. For $\mathrm{D}^{+} \rightarrow \mathrm{K}^{-} \pi^{+} \pi^{+}$, $\mathcal{P}\left(\mathrm{D}_{\mathrm{s}}^{+}\right)$is the probability to fit the $\mathrm{D}_{\mathrm{s}}^{+}$hypothesis, based on the $\mathrm{d} E / \mathrm{d} x$ measurements of the $\pi^{+}$'s and on the reconstructed masses for each of the $\mathrm{K}^{-} \mathrm{K}^{+} \pi^{+}$hypotheses 


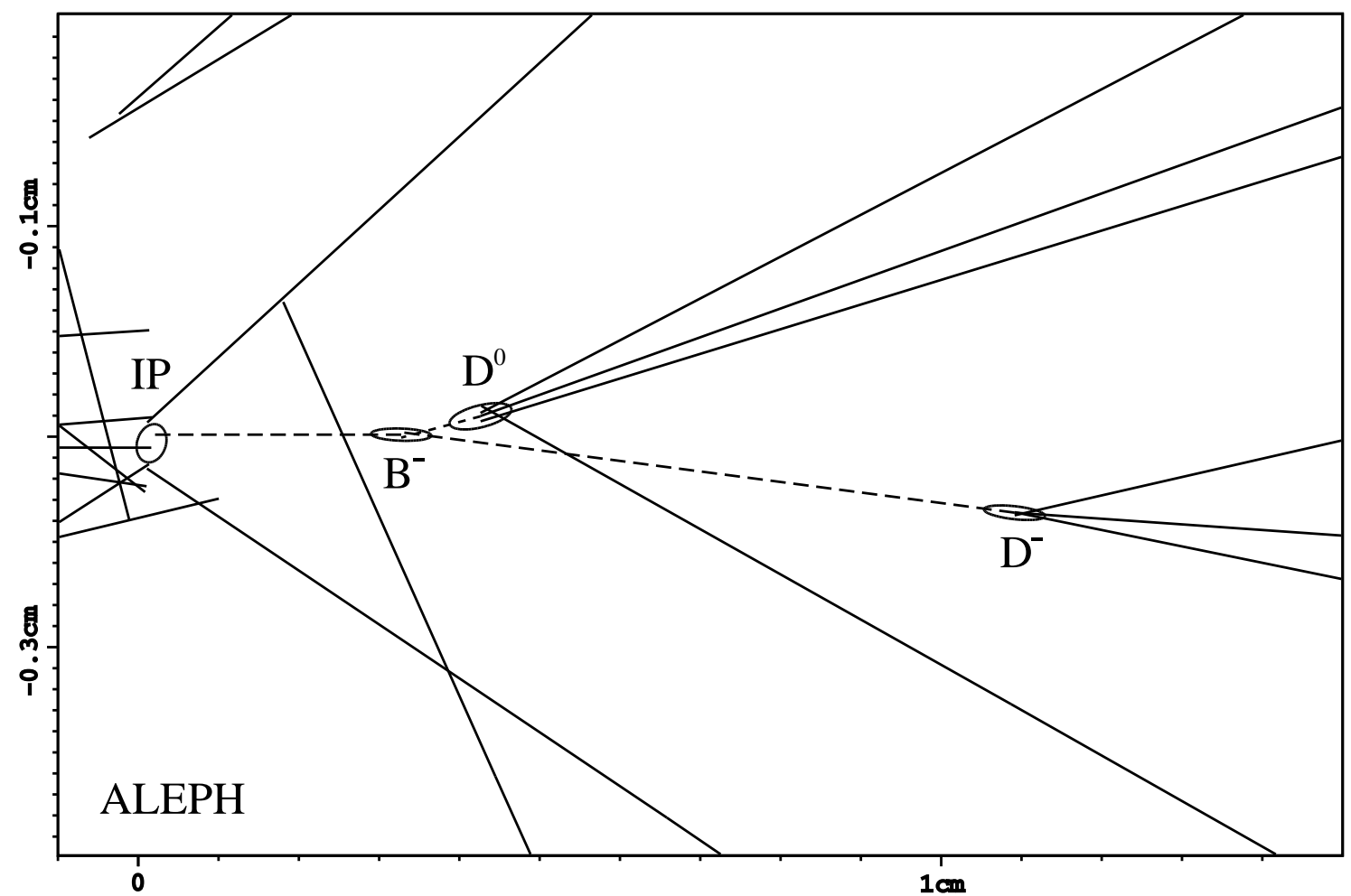

Figure 9: A closeup view of event $\mathrm{F}$ (from Table 9) near the interaction point. This event is a candidate for $\mathrm{B}^{-} \rightarrow \mathrm{D}^{*-} \mathrm{D}^{0}$ with $\mathrm{D}^{0} \rightarrow \mathrm{K}^{-} \pi^{+} \pi^{-} \pi^{+}$and $\mathrm{D}^{*-} \rightarrow \mathrm{D}^{-} \pi^{0}$ followed by $\mathrm{D}^{-} \rightarrow \mathrm{K}^{+} \pi^{-} \pi^{-}$. The error ellipses represent $2 \sigma$. 


\begin{tabular}{|c|c|c|c|}
\hline $\begin{array}{c}\text { Decay } \\
\text { channel }\end{array}$ & $\begin{array}{c}\text { Events } \\
\text { seen }\end{array}$ & $\begin{array}{c}\text { Comb. } \\
\text { background }\end{array}$ & $\begin{array}{c}90 \% \text { C.L. } \\
\text { Upper limit on } \mathcal{B}\end{array}$ \\
\hline$\overline{\mathrm{B}}^{0} \rightarrow \mathrm{D}^{0} \overline{\mathrm{D}}^{0}$ & 0 & $0.3 \pm 0.0$ & $<0.7 \%$ \\
$\overline{\mathrm{B}}^{0} \rightarrow \mathrm{D}^{0} \overline{\mathrm{D}}^{* 0}+\mathrm{D}^{* 0} \overline{\mathrm{D}}^{0}$ & 1 & $1.0 \pm 0.1$ & $<1.2 \%$ \\
$\overline{\mathrm{B}}^{0} \rightarrow \mathrm{D}^{* 0} \overline{\mathrm{D}}^{* 0}$ & 6 & $1.9 \pm 0.2$ & $<2.7 \%$ \\
\hline Sum $\overline{\mathrm{B}}^{0} \rightarrow \mathrm{D}^{* * 0} \overline{\mathrm{D}}^{(*) 0}$ & 7 & $3.2 \pm 0.3$ & $<2.7 \%$ \\
\hline
\end{tabular}

Table 10. Limits obtained on the branching fractions for the decays $\overline{\mathrm{B}}^{0} \rightarrow \mathrm{D}^{(*) 0} \overline{\mathrm{D}}^{(*) 0}$.

$V_{\mathrm{cb}} V_{\mathrm{cd}} f_{\mathrm{B}} / m_{\mathrm{B}}$, where $f_{\mathrm{B}} \simeq 200 \mathrm{MeV}$ is the $\mathrm{B}$ meson decay constant. However, it was pointed out recently [20] that final state interactions (rescattering from $\mathrm{B}^{0} \rightarrow \mathrm{D}^{(*)+} \overline{\mathrm{D}}^{(*)-}$ ) could significantly enhance this decay amplitude. For instance, the branching ratio for $\mathrm{B}^{0} \rightarrow \mathrm{D}^{0} \overline{\mathrm{D}}^{0}$ is expected to be $\mathcal{B}\left(\mathrm{B}^{0} \rightarrow \mathrm{D}^{0} \overline{\mathrm{D}}^{0}\right) \simeq 2 \times 10^{-5}[20]$. No experimental measurements of these decays currently exists and it is therefore interesting to search for them, although the statistical sensitivity expected is far from the predicted theoretical values.

The best sensitivity is obtained by using the selection criteria of the inclusive analysis and adding the requirements of no additional track at the $\mathrm{D} \overline{\mathrm{D}}$ vertex and no additional $\mathrm{K}_{\mathrm{S}}^{0}$ in the hemisphere. The $\mathrm{D}^{0} \overline{\mathrm{D}}^{0}$ mass distribution of the selected events is shown in Fig.10a. No significant excess of events over the combinatorial background is observed. The 90\% C.L. upper limits obtained on the corresponding branching fractions are given in Table 10.

\subsection{Search for the decay $B_{\mathrm{s}}^{0} \rightarrow \mathrm{D}_{\mathrm{s}}^{(*)+} \mathrm{D}_{\mathrm{s}}^{(*)-}$}

Doubly-charmed $\mathrm{B}_{\mathrm{s}}^{0}$ decays have been searched for from events with a pair of opposite sign reconstructed $D_{\mathrm{s}}$ mesons. Using the criteria described in Section 5.1, two events are observed in the whole $\mathrm{D}_{\mathrm{s}}^{+} \mathrm{D}_{\mathrm{s}}^{-}$mass spectrum, while the combinatorial background is expected to be $3.4 \pm 1.4$ events. The $\mathrm{D}_{\mathrm{s}}^{+} \mathrm{D}_{\mathrm{s}}^{-}$mass distribution of these events is shown in Fig.10b. While the low mass event is clearly compatible with the background, a candidate for a two-body decay $\mathrm{B}_{\mathrm{s}}^{0} \rightarrow \mathrm{D}_{\mathrm{s}}^{+} \mathrm{D}_{\mathrm{s}}^{-}$ is observed at $m\left(\mathrm{D}_{\mathrm{s}}^{+} \mathrm{D}_{\mathrm{s}}^{-}\right)=5.357 \pm 0.006 \mathrm{GeV} / c^{2}$, where no combinatorial background remains. The decay length of this event is $d_{\mathrm{B}}=9.5 \pm 0.2 \mathrm{~mm}$, its scaled energy is $x_{E}\left(\mathrm{D}_{\mathrm{s}}^{+} \mathrm{D}_{\mathrm{s}}^{-}\right)=0.97$ and both $\mathrm{D}$ vertices are more than 1.7 standard deviations (about $0.8 \mathrm{~mm}$ ) downstream from the $\mathrm{B}$ vertex. However, this event is also compatible with a reflection from the two-body decay $\mathrm{B}_{\mathrm{d}}^{0} \rightarrow \mathrm{D}_{\mathrm{s}}^{-} \mathrm{D}^{+}$, where the decay $\mathrm{D}^{+} \rightarrow \mathrm{K}^{-} \pi^{+} \pi^{+}$mimics a decay $\mathrm{D}_{\mathrm{s}}^{+} \rightarrow \mathrm{K}^{* 0} \mathrm{~K}^{+}\left(\mathrm{K}^{* 0} \rightarrow \mathrm{K}^{-} \pi^{+}\right)$. From the Monte Carlo and from the observed number of two-body decays $\mathrm{B}_{\mathrm{d}}^{0} \rightarrow \mathrm{D}_{\mathrm{s}}^{-} \mathrm{D}^{+}$, the expected number of reflections from $\mathrm{B}_{\mathrm{d}}^{0} \rightarrow \mathrm{D}_{\mathrm{s}}^{-} \mathrm{D}^{+}$is estimated to be 0.1 events. The following $90 \%$ confidence level upper limit on the two-body doubly-charmed $\mathrm{B}_{\mathrm{s}}^{0}$ decays is extracted

$$
\mathcal{B}\left(\mathrm{B}_{\mathrm{s}}^{0} \rightarrow \mathrm{D}_{\mathrm{s}}^{(*)-} \mathrm{D}_{\mathrm{s}}^{(*)+}\right)<21.8 \%
$$



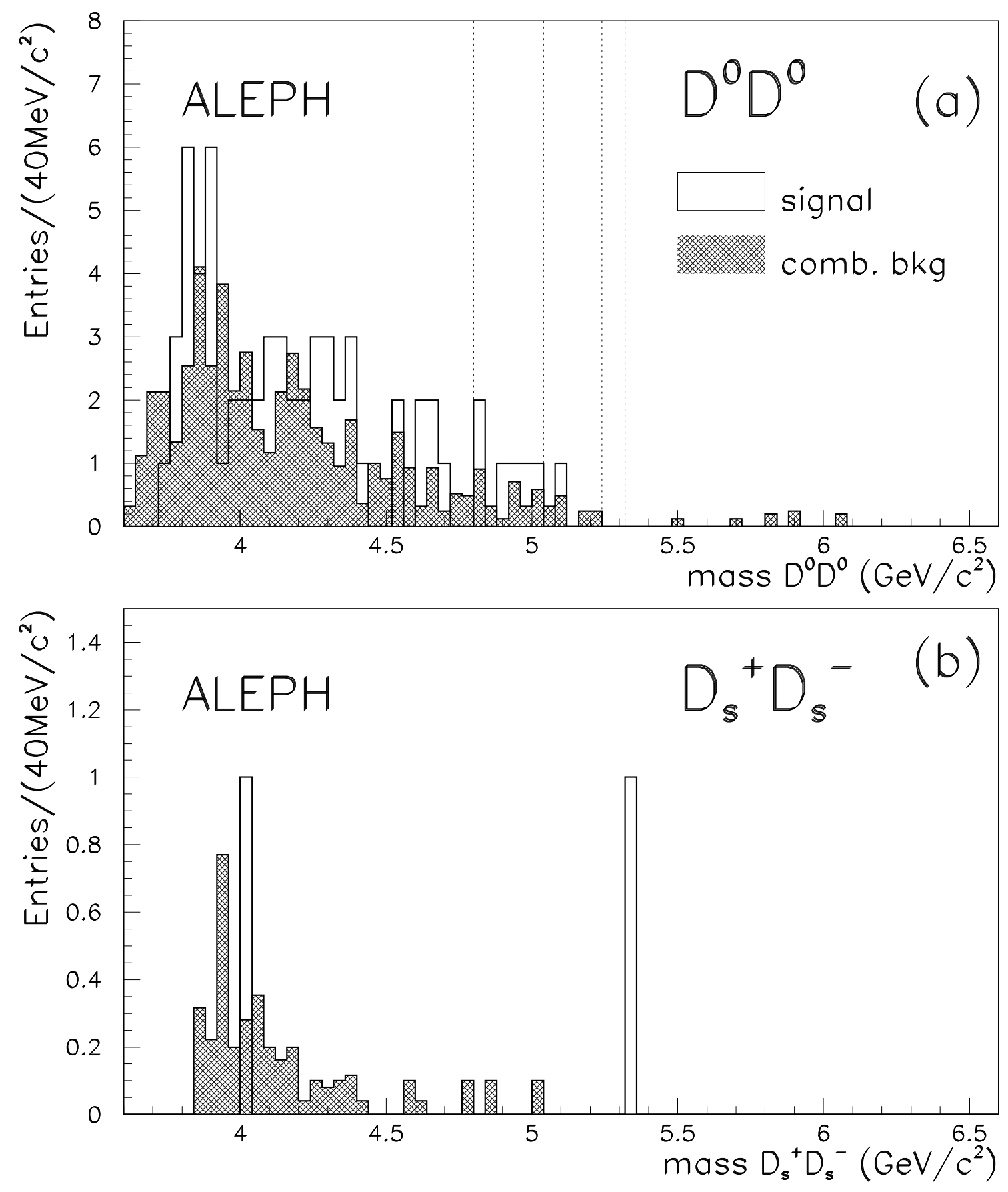

Figure 10: (a) $\mathrm{D}^{0} \overline{\mathrm{D}}^{0}$ mass distribution of the events selected in the search for decays $\mathrm{B}^{0} \rightarrow \mathrm{D}^{(*) 0} \overline{\mathrm{D}}^{(*) 0}$ (b) $\mathrm{D}_{\mathrm{s}}^{+} \mathrm{D}_{\mathrm{s}}^{-}$mass distribution of the events selected in the search for decays $\mathrm{B}_{\mathrm{s}}^{0} \rightarrow \mathrm{D}_{\mathrm{s}}^{(*)+} \mathrm{D}_{\mathrm{s}}^{(*)-}$. Unshaded histograms are signal. Shaded histograms are events in the sidebands of the $\mathrm{D}_{1}$ or the $\mathrm{D}_{2}$ mass spectra, normalised to the expected number of combinatorial background events. 


\subsection{Search for the decay $B_{c} \rightarrow D^{* \pm} D^{0}$}

The $\mathrm{D}^{* \pm} \mathrm{D}^{0}$ mass plot of Fig.4c deserves special attention since $\mathrm{D}^{* \pm} \mathrm{D}^{0}$ is a possible decay mode for the $B_{c}$ meson (by analogy with the $D_{s}$ decay to $K^{*} K$ ). However, assuming that the fraction of $B_{c}$ produced is in the range $0.6-2 \cdot 10^{-3}$ per b $\bar{b}$ pair [21], and even if the branching fraction to $\mathrm{D}^{* \pm} \mathrm{D}^{0}$ is equal to the branching fraction of the $\mathrm{D}_{\mathrm{s}}$ to $\mathrm{K}^{*} \mathrm{~K}$, the expected number of events after selection is $0.01-0.03$. One candidate event is observed in the data. The mass of this candidate is $m\left(\mathrm{D}^{*+} \overline{\mathrm{D}}^{0}\right)=6.403 \pm 0.011 \mathrm{GeV} / c^{2}$, i.e. higher than the $6.24-6.28 \mathrm{GeV} / c^{2}$ mass range predicted by theoretical models [22]. The combinatorial background expected for $m\left(\mathrm{D}^{*+} \overline{\mathrm{D}}^{0}\right)>5.4 \mathrm{GeV} / c^{2}$ is estimated to be $0.6 \pm 0.2$ events. Moreover, the reconstructed decay length of this $\mathrm{B}_{\mathrm{c}}$ candidate is $d_{\mathrm{B}}=0.1 \pm 0.1 \mathrm{~mm}$, i.e. the $\mathrm{D}^{*+} \overline{\mathrm{D}}^{0}$ vertex is compatible with the interaction point. The following $90 \%$ confidence level upper limit is extracted:

$$
\mathcal{B}\left(\mathrm{Z} \rightarrow \mathrm{B}_{\mathrm{c}} X\right) \times \mathcal{B}\left(\mathrm{B}_{\mathrm{c}} \rightarrow \mathrm{D}^{*+} \overline{\mathrm{D}}^{0}\right)<1.9 \times 10^{-3},
$$

to be compared with a theoretical expectation at the $10^{-6}$ level.

\section{Conclusion}

In this paper, a comprehensive study of all possible B meson decays into a charmed and an anticharmed meson plus anything has been performed. The inclusive branching fraction of $\mathrm{b}$ quarks to $\mathrm{D}_{\mathrm{s}} \mathrm{D}(X)$ is measured to be

$$
\mathcal{B}\left(\mathrm{b} \rightarrow \mathrm{D}_{\mathrm{s}} \mathrm{D}^{0}, \mathrm{D}_{\mathrm{s}} \mathrm{D}^{ \pm}(X)\right)=\left(13.1_{-2.2}^{+2.6}(\mathrm{stat})_{-1.6}^{+1.8}(\mathrm{syst})_{-2.7}^{+4.4}\left(\mathcal{B}_{\mathrm{D}}\right)\right) \%,
$$

in good agreement with previous measurements of the inclusive branching fraction of the $\mathrm{B}$ mesons to $D_{s}[1,2]$. For the first time, doubly-charmed $B$ decays involving no $D_{s}$ production are observed. The corresponding inclusive branching fractions are

$$
\mathcal{B}\left(\mathrm{b} \rightarrow \mathrm{D}^{0} \overline{\mathrm{D}}^{0}, \mathrm{D}^{0} \mathrm{D}^{ \pm}(X)\right)=\left(7.8_{-1.8}^{+2.0}(\text { stat })_{-1.5}^{+1.7}(\text { syst })_{-0.4}^{+0.5}\left(\mathcal{B}_{\mathrm{D}}\right)\right) \%
$$

and

$$
\mathcal{B}\left(\mathrm{b} \rightarrow \mathrm{D}^{ \pm} \mathrm{D}^{\mp}(X)\right)<0.9 \% \text { at } 90 \% \text { C.L. }
$$

Hence, as suggested in [5], a significant fraction of the doubly-charmed B decays leads to no $\mathrm{D}_{\mathrm{s}}$ production. For the average mixture of $\mathrm{b}$ hadrons produced at LEP, the sum over all the decay modes above yields:

$$
\mathcal{B}\left(\mathrm{b} \rightarrow \mathrm{D}_{\mathrm{s}} \mathrm{D}^{0}, \mathrm{D}_{\mathrm{s}} \mathrm{D}^{ \pm}, \mathrm{D}^{0} \overline{\mathrm{D}}^{0}, \mathrm{D}^{0} \mathrm{D}^{ \pm}(X)\right)=\left(20.9_{-2.8}^{+3.2}(\text { stat })_{-2.2}^{+2.5}(\mathrm{syst})_{-2.8}^{+4.5}\left(\mathcal{B}_{\mathrm{D}}\right)\right) \% .
$$

This measurement is in good agreement with the recent ALEPH measurement of the total charm rate in b events [16] $n_{c}=1.230 \pm 0.036$ (stat) \pm 0.038 (syst) $\pm 0.053\left(\mathcal{B}_{\mathrm{D}}\right)$, and with theoretical expectations [5]. 
Evidence for associated $\mathrm{K}_{\mathrm{S}}^{0}$ and $\mathrm{K}^{ \pm}$production among the $\mathrm{B} \rightarrow \overline{\mathrm{D}} \mathrm{D}(X)$ candidates is also found and 18 candidates for three-body decays $\mathrm{B} \rightarrow \overline{\mathrm{D}}^{(*)} \mathrm{D}^{(*)} \mathrm{K}$ are observed. The three-body decay branching fraction, averaged over $\mathrm{B}_{\mathrm{d}}^{0}$ and $\mathrm{B}^{ \pm}$, is measured to be

$$
\mathcal{B}\left(\mathrm{B} \rightarrow \overline{\mathrm{D}}^{(*)} \mathrm{D}^{(*)} \mathrm{K}\right)=\left(7.1_{-1.5}^{+2.5}(\text { stat })_{-0.8}^{+0.9}(\text { syst }) \pm 0.5\left(\mathcal{B}_{\mathrm{D}}\right)\right) \%
$$

Compared to the inclusive $\mathrm{b}$ results above, scaled by a factor $1 / 2 \mathrm{f}_{\mathrm{B}_{\mathrm{d}}^{0}}=1.3$ to account for $\mathrm{b} \rightarrow \overline{\mathrm{B}}^{0}, \mathrm{~B}^{-}$, one sees that the three-body decays $\mathrm{B} \rightarrow \overline{\mathrm{D}}^{(*)} \mathrm{D}^{(*)} \mathrm{K}$ are a large part of the inclusive doubly-charmed $\mathrm{B} \rightarrow \overline{\mathrm{D}} \mathrm{D}(X)$ decays . No evidence for decays $\mathrm{B} \rightarrow \overline{\mathrm{D}}^{(*)} \mathrm{D}_{\mathrm{s} 1}^{+}(2535)$ is found.

Semi-exclusive doubly-charmed $B$ decays involving a $D_{s}$ meson in the final state have also been studied. Through the reconstruction of both the $\overline{\mathrm{D}}$ and the $\mathrm{D}_{\mathrm{s}}$, this analysis clearly establishes that the low $x_{E} D_{\mathrm{s}}$ production observed at the $\Upsilon(4 S)$ is indeed due to decays $\mathrm{B}^{0}, \mathrm{~B}^{+} \rightarrow \overline{\mathrm{D}}^{(*)} \mathrm{D}_{\mathrm{s}}^{+} X$. For the first time, some candidates for completely reconstructed decays $\mathrm{B}^{0}, \mathrm{~B}^{+} \rightarrow \overline{\mathrm{D}}^{(*)} \mathrm{D}_{\mathrm{s}}^{+} n \pi^{ \pm}(n \geq 1)$ are also observed. A measurement of the branching fraction for many-body decays $\mathrm{B}^{0}, \mathrm{~B}^{+} \rightarrow \overline{\mathrm{D}}^{(*)} \mathrm{D}_{\mathrm{s}}^{+} X$ is performed, leading to

$$
\mathcal{B}\left(\mathrm{B} \rightarrow \mathrm{D}_{\mathrm{s}}^{(*) \pm} \mathrm{D}^{(*)} X\right)=\left(9.4_{-3.1}^{+4.0}(\text { stat })_{-1.8}^{+2.2}(\text { syst })_{-1.6}^{+2.6}\left(\mathcal{B}_{\mathrm{D}}\right)\right) \% \text {. }
$$

The branching fraction of $\mathrm{B}^{0}$ and $\mathrm{B}^{+}$mesons into doubly-charmed two-body decay modes is also measured and gives

$$
\mathcal{B}\left(\mathrm{B} \rightarrow \mathrm{D}_{\mathrm{s}}^{(*)+} \overline{\mathrm{D}}^{(*)}\right)=\left(5.6_{-1.5}^{+2.1}(\text { stat })_{-0.8}^{+0.9}(\text { syst })_{-1.1}^{+1.9}\left(\mathcal{B}_{\mathrm{D}}\right)\right) \%,
$$

in good agreement with previous measurements of the same quantity [1, 2].

Finally, two candidates for the Cabibbo suppressed decay $\mathrm{B}_{\mathrm{d}}^{0} \rightarrow \mathrm{D}^{*+} \mathrm{D}^{*-}$ are observed. The corresponding branching fraction is measured to be

$$
\mathcal{B}\left(\overline{\mathrm{B}}_{\mathrm{d}}^{0} \rightarrow \mathrm{D}^{*+} \mathrm{D}^{*-}\right)=\left(0.23_{-0.12}^{+0.19}(\text { stat }) \pm 0.04(\text { syst }) \pm 0.02\left(\mathcal{B}_{\mathrm{D}}\right)\right) \% .
$$

One candidate for the Cabibbo suppressed decay $\mathrm{B}^{-} \rightarrow \mathrm{D}^{*-} \mathrm{D}^{0}$, with both $\mathrm{D}$ vertices well separated from the reconstructed $\mathrm{B}$ decay point, is also observed.

\section{Acknowledgements}

We wish to thank our colleagues from the accelerator divisions for the successful operation of LEP. We are indebted to the engineers and technicians in all our institutions for their contribution to the excellent performance of ALEPH. Those of us from non-member countries thank CERN for its hospitality. 


\section{Appendix: $\mathrm{D} \overline{\mathrm{D}} \mathrm{K}^{ \pm}$and $\mathrm{D} \overline{\mathrm{D}} \mathrm{K}_{\mathrm{S}}^{0}$ event properties}

\begin{tabular}{|l|c|c|c|c|c|c|c|c|c|}
\hline Run & 12049 & 16176 & 16744 & 26062 & 26478 & 26814 & 26856 & 28490 & 36630 \\
Event & 2539 & 6779 & 1804 & 3422 & 7624 & 5048 & 1266 & 6843 & 5090 \\
\hline $\mathrm{B}$ type & $\overline{\mathrm{B}}^{0}$ & $\overline{\mathrm{B}}^{0}$ & $\mathrm{~B}^{+}$ & $\mathrm{B}^{-}$ & $\mathrm{B}^{+}$ & $\mathrm{B}^{0}$ & $\mathrm{~B}^{0}$ & $\mathrm{~B}^{+}$ & $\overline{\mathrm{B}}^{0}$ \\
$\mathrm{D}_{1}($ from b) & $\mathrm{D}^{*+}$ & $\mathrm{D}^{+}$ & $\overline{\mathrm{D}}^{0}$ & $\mathrm{D}^{0}$ & $\mathrm{D}^{*-}$ & $\mathrm{D}^{-}$ & $\mathrm{D}^{-}$ & $\mathrm{D}^{-}$ & $\mathrm{D}^{+}$ \\
$\mathrm{D}_{2}($ from W) & $\overline{\mathrm{D}}^{0}$ & $\overline{\mathrm{D}}^{0}$ & $\mathrm{D}^{0}$ & $\overline{\mathrm{D}}^{0}$ & $\mathrm{D}^{+}$ & $\mathrm{D}^{0}$ & $\mathrm{D}^{0}$ & $\mathrm{D}^{*+}$ & $\overline{\mathrm{D}}^{0}$ \\
$\mathrm{~K}$ & $\mathrm{~K}^{-}$ & $\mathrm{K}^{-}$ & $\mathrm{K}^{+}$ & $\mathrm{K}^{-}$ & $\mathrm{K}^{+}$ & $\mathrm{K}^{+}$ & $\mathrm{K}^{+}$ & $\mathrm{K}^{+}$ & $\mathrm{K}^{-}$ \\
Diag. Type & $\mathrm{E}$ & $\mathrm{E}$ & $\mathrm{IE}$ & $\mathrm{IE}$ & $\mathrm{I}$ & $\mathrm{E}$ & $\mathrm{E}$ & $\mathrm{I}$ & $\mathrm{E}$ \\
$x_{E}(\mathrm{DDK})$ & 0.81 & 0.64 & 0.61 & 0.60 & 0.68 & 0.57 & 0.91 & 0.69 & 0.72 \\
$p\left(\mathrm{D}_{1}\right)$ & 14.5 & 13.6 & 11.8 & 13.2 & 6.8 & 13.9 & 14.4 & 13.9 & 14.3 \\
$p\left(\mathrm{D}_{2}\right)$ & 19.3 & 10.3 & 14.7 & 10.3 & 17.9 & 9.7 & 15.0 & 12.5 & 12.0 \\
$p(\mathrm{~K})$ & 2.8 & 4.8 & 0.8 & 3.5 & 5.8 & 2.3 & 11.7 & 4.6 & 6.2 \\
$\chi_{K}(\mathrm{~K})$ & +0.7 & +0.4 & 0.4 & -0.3 & +0.1 & +0.1 & +0.0 & -2.2 & +0.6 \\
$\chi_{\pi}(\mathrm{K})$ & -1.0 & -0.8 & 2.3 & -2.2 & -2.2 & -1.7 & -2.2 & -4.2 & -1.6 \\
$m\left(\mathrm{D}_{1} \mathrm{D}_{2}\right)$ & $(4.41)$ & $(4.06)$ & 3.79 & $4.50^{*}$ & 4.61 & $(4.30)$ & $(4.37)$ & $4.08^{*}$ & $(3.99)$ \\
$m\left(\mathrm{D}_{1} \mathrm{~K}\right)$ & $(2.958)$ & $(3.056)$ & $(3.063)$ & $(2.675)^{*}$ & $(2.883)$ & $(3.165)$ & $(2.948)$ & $(2.926)^{*}$ & $(3.141)$ \\
$m\left(\mathrm{D}_{2} \mathrm{~K}\right)$ & 2.710 & 2.793 & 3.335 & 2.377 & $(2.494)$ & 2.531 & 2.695 & $(2.888)$ & 2.635 \\
$m\left(\mathrm{D}_{1} \mathrm{D}_{2} \mathrm{~K}\right)$ & 5.27 & 5.14 & 5.26 & 5.08 & 5.29 & 5.26 & 5.27 & 5.09 & 5.05 \\
$d_{\mathrm{B}}(\mathrm{mm})$ & 3.3 & 3.2 & 1.9 & 3.7 & 1.4 & 1.0 & 7.3 & 6.9 & 8.2 \\
$\sigma_{d_{\mathrm{B}}}$ & 0.2 & 0.1 & 0.4 & 0.2 & 0.3 & 0.2 & 0.3 & 0.2 & 0.4 \\
$d_{\mathrm{BD}_{1}} / \sigma$ & 7.2 & 6.8 & 1.1 & +9.8 & +2.5 & +2.2 & +30.4 & +3.1 & -1.2 \\
$d_{\mathrm{BD}_{2} / \sigma}$ & 4.0 & 4.0 & 5.3 & +2.1 & +1.8 & +1.2 & +4.3 & -0.2 & 0.3 \\
$\chi_{\pi}\left(\mathrm{K}\right.$ from $\left.\mathrm{D}_{1}\right)$ & -1.7 & -2.5 & -3.0 & -1.0 & -0.1 & -1.7 & -1.6 & -0.9 & -0.4 \\
$\chi_{\pi}\left(\mathrm{K}\right.$ from $\left.\mathrm{D}_{2}\right)$ & -1.8 & -2.4 & -1.9 & - & -1.2 & -0.9 & -2.1 & -2.5 & - \\
\hline
\end{tabular}

Table 11. Properties of the 5 fully reconstructed and the 4 partially reconstructed $\mathrm{D}^{(*)} \mathrm{D}^{(*)} \mathrm{K}^{ \pm}$ events. () means resonance impossible in $c \bar{c}\left[m\left(\mathrm{D}_{1} \mathrm{D}_{2}\right)\right]$ or in $\mathrm{c} \overline{\mathrm{s}}\left[m\left(\mathrm{D}_{1} \mathrm{~K}\right), m\left(\mathrm{D}_{2} \mathrm{~K}\right)\right]$, due to the electric charge. The diagram types E, I and EI mean external, internal or both spectator diagrams. The $*$ means $150 \mathrm{MeV} / c^{2}$ must be added to obtain the fully reconstructed event; it is quoted only for events where the partially reconstructed $\mathrm{D}^{*}$ is unambiguous. 


\begin{tabular}{|c|c|c|c|c|c|c|c|c|c|}
\hline Run & 15066 & 15931 & 16249 & 23223 & 27804 & 29425 & 36643 & 37192 & 37789 \\
\hline Event & 499 & 5619 & 3332 & 5757 & 742 & 7168 & 2440 & 9758 & 15771 \\
\hline B type & $\mathrm{B}^{-}$ & $\mathrm{B}^{-}$ & $\mathrm{B}^{0}, \overline{\mathrm{B}}^{0}$ & $\mathrm{~B}^{+}$ & $\mathrm{B}^{0}, \overline{\mathrm{B}}^{0}$ & $\mathrm{~B}^{0}, \overline{\mathrm{B}}^{0}$ & $\mathrm{~B}^{0}, \overline{\mathrm{B}}^{0}$ & $\mathrm{~B}^{+}$ & $\mathrm{B}^{0}, \overline{\mathrm{B}}^{0}$ \\
\hline $\mathrm{D}_{1}$ type & $\mathrm{D}^{0}$ & $\mathrm{D}^{0}$ & $\mathrm{D}^{+}$ & $\overline{\mathrm{D}}^{0}$ & $\mathrm{D}^{*-}$ & $\mathrm{D}^{+}$ & $\mathrm{D}^{*-}$ & $\overline{\mathrm{D}}^{0}$ & $\overline{\mathrm{D}}^{0}$ \\
\hline $\mathrm{D}_{2}$ type & $\mathrm{D}^{*-}$ & $\mathrm{D}^{*-}$ & $\mathrm{D}^{*-}$ & $\mathrm{D}^{+}$ & $\mathrm{D}^{*+}$ & $\mathrm{D}^{*-}$ & $\mathrm{D}^{+}$ & $\mathrm{D}^{+}$ & $\mathrm{D}^{0}$ \\
\hline Diag. type & $\mathrm{E}$ & $\mathrm{E}$ & $\mathrm{IE}$ & $\mathrm{E}$ & $\mathrm{IE}$ & $\mathrm{IE}$ & $\mathrm{IE}$ & $\mathrm{E}$ & $\mathrm{I}$ \\
\hline$x_{E}\left(\mathrm{D}_{1} \mathrm{D}_{2} \mathrm{~K}^{0}\right)$ & 0.74 & 0.75 & 0.75 & 0.94 & 0.75 & 0.89 & 0.73 & 0.74 & 0.77 \\
\hline$p\left(\mathrm{D}_{1}\right)$ & 12.1 & 14.1 & 13.6 & 25.8 & 16.2 & 20.1 & 17.6 & 17.3 & 8.8 \\
\hline$p\left(\mathrm{D}_{2}\right)$ & 16.8 & 17.3 & 11.8 & 13.7 & 12.3 & 14.8 & 12.6 & 13.4 & 20.3 \\
\hline$p\left(\mathrm{~K}^{0}\right)$ & 4.7 & 2.8 & 8.3 & 3.7 & 5.2 & 5.6 & 2.6 & 3.3 & 6.5 \\
\hline$m\left(\mathrm{D}_{1} \mathrm{D}_{2}\right)$ & $(4.51)^{*}$ & $(4.56)$ & 4.35 & 4.72 & 4.57 & 4.26 & 4.69 & $(4.17)^{*}$ & 4.63 \\
\hline$m\left(\mathrm{D}_{1} \mathrm{~K}^{0}\right)$ & $(2.693)^{*}$ & $(2.655)$ & 2.619 & $(2.466)$ & 2.630 & 2.754 & 2.607 & $(2.903)^{*}$ & $(2.810)$ \\
\hline$m\left(\mathrm{D}_{2} \mathrm{~K}^{0}\right)$ & 2.585 & 2.795 & 3.139 & 2.603 & 2.968 & 3.206 & 2.619 & 2.702 & $(2.460)$ \\
\hline$m\left(\mathrm{D}_{1} \mathrm{D}_{2} \mathrm{~K}^{0}\right)$ & 5.147 & 5.280 & 5.273 & 5.289 & 5.298 & 5.303 & 5.279 & 5.088 & 5.309 \\
\hline$d_{\mathrm{B}}(\mathrm{mm})$ & 6.2 & 2.2 & 2.7 & 9.1 & 19 & 6.5 & 2.7 & 5.0 & 3.1 \\
\hline$\sigma_{d_{\mathrm{B}}}$ & 0.2 & 0.2 & 0.3 & 0.2 & 0.5 & 1.5 & 0.2 & 0.2 & 0.2 \\
\hline$d_{\mathrm{BD}_{1}} / \sigma$ & +0.8 & +0.4 & +1.8 & +0.8 & +0.7 & +4.9 & +5.5 & +2.7 & -0.8 \\
\hline$d_{\mathrm{BD}_{2}} / \sigma$ & +5.0 & +8.1 & +4.2 & +12.4 & +0.6 & -1.1 & +2.3 & +1.0 & +0.3 \\
\hline$\chi_{\pi}\left(\mathrm{K}\right.$ from $\left.\mathrm{D}_{1}\right)$ & -2.7 & -2.3 & -1.0 & -1.7 & - & -2.8 & -2.9 & -2.7 & -3.0 \\
\hline$\chi_{\pi}\left(\mathrm{K}\right.$ from $\left.\mathrm{D}_{2}\right)$ & -2.9 & -2.4 & -0.4 & -2.7 & -0.4 & -2.0 & -1.5 & -1.9 & -2.4 \\
\hline
\end{tabular}

Table 12. Properties of the 7 fully reconstructed and the 2 partially reconstructed $\mathrm{D}^{(*)} \mathrm{D}^{(*)} \mathrm{K}^{0}$ events. () means resonance impossible in $c \bar{c}\left[m\left(D_{1} \mathrm{D}_{2}\right)\right]$ or in $c \overline{\mathrm{s}}\left[m\left(\mathrm{D}_{1} \mathrm{~K}\right), m\left(\mathrm{D}_{2} \mathrm{~K}\right)\right]$, due to the electric charge. The $*$ means $150 \mathrm{MeV} / c^{2}$ must be added to obtain the fully reconstructed event; it is quoted only for events where the partially reconstructed $\mathrm{D}^{*}$ is unambiguous.

\section{References}

[1] ARGUS collab., H.Albrecht et al., Z. Phys C54 (1992) 1.

[2] CLEO collab., D.Gibaut et al., Phys.Rev. D53 (1996) 4734.

[3] T.Browder, 'Hadronic decays and lifetimes of B and D mesons', proceedings of the 1996 Warsaw ICHEP conference, Z.Ajduk and A.K.Wroblewski Eds, World Scientific (1997) p1139.

[4] I.I.Bigi, B.Blok, M.Shifman and A.Vainshtein, Phys. Lett. B323 (1994) 408.

[5] G.Buchalla, I.Dunietz and H.Yamamoto, Phys.Lett. B364 (1995) 188. 
[6] CLEO collab., CLNS 97/1516, submitted to Phys. Rev. Lett.

[7] ALEPH collab., D.Decamp et al., Nucl. Instr. and Meth. A294 (1990) 121.

[8] ALEPH collab., D.Buskulic et al., Nucl. Instr. and Meth. A360 (1995) 481.

[9] ALEPH collab., D.Decamp et al., Z. Phys C53 (1992) 1.

[10] ALEPH collab., D.Buskulic et al., Phys. Lett. B295 (1992) 174.

[11] T.Sjöstrand, Computer Physics Commun. 82 (1994) 74.

[12] C.Peterson, D.Schlatter, I.Schmitt and P.M.Zerwas, Phys. Rev. D27 (1983) 105.

[13] The LEP experiments, Nucl. Instr. and Meth. A378 (1996) 101.

[14] CLEO collab., D.M.Asner et al., Phys.Rev.Lett. 79 (1997) 803.

[15] Particle Data Group, R.M.Barnett et al., Phys. Rev. D54 (1996).

[16] ALEPH collaboration, D.Buskulic et al., Phys. Lett. B388 (1996) 648.

[17] OPAL collab., R.Akers et al., Phys.Lett. B353 (1995) 595.

[18] H.J.Lipkin and A.I.Sanda, Phys. Lett. B201 (1988) 541.

[19] I.Dunietz, Fermilab report, FERMILAB-PUB-96/104-T, June 1996 [hep-ph/9606247].

[20] B.Blok, M.Gronau and J.L.Rosner, Phys. Rev. Lett. 78 (1997) 3999.

[21] V.V.Kiselev, A.K.Likhoded and M.V.Shevlyagin, Phys. Atom. Nucl. 57 (1994) 689.

[22] A.Martin, 'Heavy flavours and high energy collisions in the TeV range', A.Ali and L.Cifarelli eds, Plenum press (1989) p141; E.Eichten and C.Quigg, Phys. Rev D49 (1994) 5845 . 